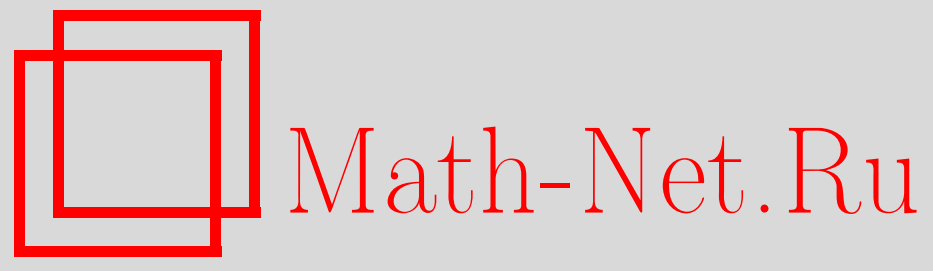

А. Ф. Заусаев, Сопоставление координат больших планет, Луны и Солнца, полученных на основе нового принципа взаимодействия и банка данных DE405, Вестн. Сам. гос. техн. ун-та. Сер. Физ.мат. науки, 2016, номер 1, 121-148

DOI: https://doi.org/10.14498/vsgtu1458

Использование Общероссийского математического портала MathNet.Ru подразумевает, что вы прочитали и согласны с пользовательским соглашением

http://www.mathnet.ru/rus/agreement

Параметры загрузки:

IP: 54.162 .127 .20

26 апреля 2023 г., 15:51:18

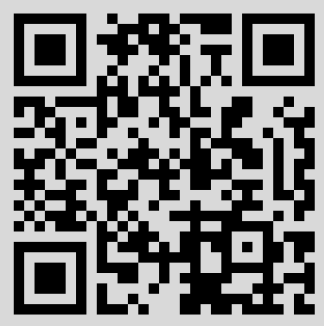


УДК 521.1, 521.4

\title{
СОПОСТАВЛЕНИЕ КООРДИНАТ БОЛЬШИХ ПЛАНЕТ, ЛУНЫ И СОЛНЦА, ПОЛУЧЕННЫХ НА ОСНОВЕ НОВОГО ПРИНЦИПА ВЗАИМОДЕЙСТВИЯ И БАНКА ДАННЫХ DE405
}

\section{A. Ф. Заусаев}

Самарский государственный технический университет, Россия, 443100, Самара, ул. Молодогвардейская, 244.

\begin{abstract}
Аннотация
В данной статье проведено сравнение координат и элементов орбит больших планет, Луны и Солнца, полученных на основе нового принципа взаимодействия и банка данных DE405. Под окружающим пространством можно понимать физический вакуум. Гравитация рассматривается как результат взаимодействия окружающего пространства с движущимися материальными телами. Тяготение объясняется свойством сжатия пространства относительно движущихся материальных тел. Получены дифференциальные уравнения движения больших планет, Луны и Солнца. Следует отметить, что система дифференциальных уравнений не содержит явно масс тел и силовых взаимодействий, кроме того, Земля рассматривается как сфероид. Путем численного интегрирования уравнений движения вычислены координаты Луны, Солнца больших планет и оскулирующие элементы орбит внутренних планет на интервале времени 1602-2193 гг. Результаты вычислений сопоставлены с координатами и элементами орбит, определенными по данным координат и скоростей DE405. Показано, что в отличие от механики Ньютона и релятивистских уравнений движения, координаты больших планет Луны и Солнца, основанные на решении новой системы дифференциальных уравнений, удовлетворительно согласуются с координатами этих объектов, вычисленных с помощью банка данных DE405. Полученные уравнения не содержат членов, учитывающих несферичность Земли и Луны, являясь при этом нерелятивистскими уравнениями. На основе исследований сделаны следующие выводы: полученные дифференциальные уравнения движения удовлетворительно описывают движение больших планет, Луны и Солнца на интервале времени 600 лет; они значительно проще и точнее дифференциальных уравнений, учитывающих релятивистские эффекты.
\end{abstract}

Ключевые слова: элементы орбит, численное интегрирование, дифференциальные уравнения движения.

(C) 2016 Самарский государственный технический университет.

\section{Образец для цитирования}

Заусаев А. Ф. Сопоставление координат больших планет, Луны и Солнца, полученных на основе нового принципа взаимодействия и банка данных DE405 // Becmн. Caм. гос. техн. ун-та. Сер. Физ.-мат. науки, 2016. Т. 20, № 1. С. 121-148. doi: 10.14498/vsgtu1458. Сведения об авторе

Анатолий Федорович Заусаев (д.ф.-м.н., проф.; zausaev_af@mail.ru), профессор, каф. прикладной математики и информатики. 
Введение. Небесная механика всегда являлась образцовым примером научного достижения в прогнозировании движения небесных тел с величайшей точностью. Построение небесной механики, как и любой науки, например геометрии, основывается на ряде основополагающих аксиом. В основе классической небесной механики лежит закон всемирного тяготения Ньютона и три закона (аксиомы) движения.

На основании закона всемирного тяготения и трех законов движения получены дифференциальные уравнения движения в задаче $n$ тел в прямоугольных координатах с началом в центре масс всей системы $n$ материальных точек, которые имеют следующий вид $[1,2]$ :

$$
\begin{aligned}
\frac{d^{2} X}{d t^{2}} & =\sum_{i} k^{2} m_{i}\left(\frac{X_{i}-X}{\Delta_{i}^{3}}\right), \\
\frac{d^{2} Y}{d t^{2}} & =\sum_{i} k^{2} m_{i}\left(\frac{Y_{i}-Y}{\Delta_{i}^{3}}\right), \\
\frac{d^{2} Z}{d t^{2}} & =\sum_{i} k^{2} m_{i}\left(\frac{Z_{i}-Z}{\Delta_{i}^{3}}\right),
\end{aligned}
$$

где $\Delta_{i}^{2}=\left(X_{i}-X\right)^{2}+\left(Y_{i}-Y\right)^{2}+\left(Z_{i}-Z\right)^{2}$, а $m_{i}, X_{i}, Y_{i}, Z_{i}$ - массы и барицентрические координаты возмущающих тел.

[В начале 1850 г. французским ученым Леверье была построена теория движения Солнца и семи больших планет (Меркурий-Нептун) относительно Земли. Результатом его труда явилось доказательство невозможности представления наблюдений прохождения Меркурия по диску Солнца на основе ньютоновской динамики любой системой оскулирующих элементов и масс известных ему планет $[3,4]$.

В конце XIX столетия Ньюком и Тиссеран пришли к выводу о существовании трех основных расхождений ньютоновской теории с астрономическими наблюдениями. Эти отклонения для векового движения перигелия Меркурия составляли $41^{\prime \prime}-43^{\prime \prime}$ (секунды дуги), для Венеры - 10" и Mapca - 8".

Наряду с установленными эмпирическими трудностями стали все более выявляться и обсуждаться трудности, связанные с феноменологическим характером ньютоновской теории. Различные затруднения вызывали у ученых сомнения в точности закона Ньютона и делались многочисленные попытки внести поправки в точную формулу закона тяготения.] ${ }^{1}$ Однако эти попытки улучшения закона всемирного тяготения оставались безуспешными. Самым существенным недостатком небесной механики Ньютона являлся принцип дальнодействия, допускающий возможность непосредственного действия данного тела на сколь угодно большом расстоянии без посредства промежуточной среды. Гравитации приписывается бесконечно большая скорость распространения, что противоречит основным представлениям современной физики.

Отказ от принципа дальнодействия, считавшегося слабой стороной небесной механики Ньютона, предпринимался неоднократно. Наиболее глубокие исследования в этом направлении были проведены Лапласом и Пуанкаре [6].

\footnotetext{
${ }^{1}$ Текст воспроизводится по работе автора [5].
} 
Однако проведенные ими исследования не привели к положительным результатам.

В первом случае возникали неоправданно большие изменения большой полуоси небесных тел, не согласующиеся с наблюдениями, на что было указано Лапласом. Во втором случае, хотя теория Пуанкаре позволяет устранить дальнодействие и согласовать закон тяготения с принципами СТО (специальная теория относительности), она не является более точной и удобной с точки зрения практического применения по сравнению с механикой Ньютона.

Следующим шагом в развитии релятивистской теории тяготения является созданная Эйнштейном общая теория относительности. В основе общей теории относительности лежат следующие гипотезы [6,7].

I. Пространство общей теории относительности должно представлять собой псевдориманово четырехмерное пространство с метрикой

$$
d s^{2}=g_{\alpha \beta} d x^{i} d x^{j},
$$

где $d s-$ расстояние; $g_{\alpha \beta}$ - метрические тензоры; $d x^{i}, d x^{j}$ - дифференциалы от криволинейных координат; греческие индексы пробегают значения $0,1,2,3$, латинские - $1,2,3$ [7, с. 33].

II. Тензор энергии-импульса $T^{i j}$ накладывается на пространство событий в качестве дополнительной конструкции, при этом принимается, что тензор энергии-импульса вытекает из самой псевдоримановой геометрии этого пространства и определяется формулой

$$
-\chi T_{i j}=R_{i j}-\frac{1}{2} R g_{i j}
$$

где $\chi$ - некоторая положительная постоянная; $T_{i j}$ - тензор энергии-импульса с опущенными (при помощи метрического тензора $g_{i j}$ ) индексами; $R_{i j}$ - тензор Риччи; $R$-скалярная кривизна в псевдоримановом пространстве событий [8, с. 621].

Уравнения (2) - уравнения поля, определяемые десятью нелинейными дифференциальными уравнениями в частных производных второго порядка гиперболического вида. Они определяют десять неизвестных функций: шесть компонент метрического тензора $g_{\alpha \beta}$ (четыре компоненты остаются произвольными), три составляющие пространственной скорости $v^{i}$ вещества и плотности масс $\rho$.

Чтобы получить решение в какой-нибудь координатной системе, необходимо добавить к уравнениям поля четыре нетензорных уравнения, которые обуславливают выбор координат. В задачах релятивистской небесной механики преимущественно используются гармонические координаты, определяемые уравнениями

$$
\frac{\partial\left(\sqrt{-g} g^{\alpha \beta}\right)}{\partial x^{\beta}}=0,
$$

где $g$ означает определитель, составленный из компонент метрического тензора.

Дифференциальные уравнения движения небесного тела в гелиоцентрической системе координат с учетом релятивистских эффектов от Солнца имеют следующий вид [7]: 


$$
\begin{aligned}
\frac{d^{2} X}{d t^{2}} & =-k^{2}(1+m) \frac{X}{r^{3}}+\sum_{i} k^{2} m_{i}\left(\frac{X_{i}-X}{\Delta_{i}^{3}}-\frac{X_{i}}{r_{i}^{3}}\right)+ \\
& +\frac{k^{2}}{c^{2}}\left[(4-2 \alpha) \frac{k^{2}}{r^{4}} X-(1+\alpha) \frac{\dot{r}^{2}}{r^{3}} X+3 \alpha \frac{(X \dot{X})^{2}}{r^{5}} X+(4-2 \alpha) \frac{(X \dot{X})}{r^{3}} \dot{X}\right],
\end{aligned}
$$

где $X$-матрица-столбец с элементами $x, y, z ; X_{i}$-матрица-столбец с элементами $x_{i}, y_{i}, z_{i} ; m, x, y, z$ - масса и гелиоцентрические координаты возмущаемого тела; $m_{i}, x_{i}, y_{i}, z_{i}$ - массы и гелиоцентрические координаты больших планет; $r, \Delta_{i}, r_{i}$ - расстояния, вычисляемые по формулам

$$
r^{2}=x^{2}+y^{2}+z^{2} . \quad \Delta_{i}^{2}=\left(x_{i}-x\right)^{2}+\left(y_{i}-y\right)^{2}+\left(z_{i}-z\right)^{2} . \quad r_{i}^{2}=x_{i}^{2}+y_{i}^{2}+z_{i}^{2} ;
$$

$\dot{X}$ - матрица-столбец с элементами $\dot{x}, \dot{y}, \dot{z} ; k$ - постоянная Гаусса; $c$ - скорость света; $\alpha$-параметр, характеризующий выбор системы координат. Случай $\alpha=1$ соответствует стандартным координатам, случай $\alpha=0$-гармоническим координатам.

[Дифференциальные уравнения движения в барицентрической системе координат с учетом ньютоновских и шварцшильдовских членов, обусловленных взаимным влиянием Солнца и планет, имеют следующий вид [9]:

$$
\begin{gathered}
\ddot{\mathbf{r}}_{i}=\sum_{j \neq i} \frac{\mu_{j}\left(\mathbf{r}_{j}-\mathbf{r}_{i}\right)}{r_{i j}^{3}}\left\{1-\frac{2(\beta+\gamma)}{c^{2}} \sum_{k \neq i} \frac{\mu_{k}}{r_{i k}}-\frac{2 \beta-1}{c^{2}} \sum_{k \neq j} \frac{\mu_{k}}{r_{j k}}+\right. \\
+\gamma\left(\frac{v_{i}}{c}\right)^{2}+(1+\gamma)\left(\frac{v_{j}}{c}\right)^{2}-\frac{2(1+\gamma)}{c^{2}} \dot{\mathbf{r}_{i}} \cdot \dot{\mathbf{r}_{j}-} \\
\left.\quad-\frac{3}{2 c^{2}}\left[\frac{\left(\mathbf{r}_{i}-\mathbf{r}_{j}\right) \cdot \dot{\mathbf{r}_{i}}}{r_{i j}}\right]^{2}+\frac{1}{2 c^{2}}\left(\mathbf{r}_{j}-\mathbf{r}_{i}\right) \cdot \ddot{\mathbf{r}_{j}}\right\}+ \\
+\frac{1}{c^{2}} \sum_{j \neq i} \frac{\mu_{j}}{r_{i j}^{3}}\left\{\left[\left(\mathbf{r}_{i}-\mathbf{r}_{j}\right)\right] \times\left[(2+2 \gamma) \dot{\mathbf{r}_{i}}-(1+2 \gamma) \dot{\mathbf{r}_{j}}\right]\right\} \cdot\left(\dot{\mathbf{r}}_{i}-\dot{\mathbf{r}}_{j}\right)+ \\
+\frac{3+4 \gamma}{2 c^{2}} \sum_{j \neq i} \frac{\mu_{j} \ddot{\mathbf{r}_{j}}}{r_{i j}}+\sum_{m=1}^{N} \frac{\mu_{m}\left(\mathbf{r}_{m}-\mathbf{r}_{j}\right)}{r_{i m}^{3}},
\end{gathered}
$$

где $\mathbf{r}_{i}, \dot{\mathbf{r}}_{i}, \ddot{\mathbf{r}}{ }_{i}$ - векторы координат, скоростей, ускорений в барицентрической системе координат $i$-того тела; $\mu_{j}=k^{2} m_{j}$, где $k^{2}$ - гравитационная постоянная и $m_{j}$-масса $j$-того тела; $r_{i j}=\left|\mathbf{r}_{j}-\mathbf{r}_{i}\right| ; \beta$ и $\gamma$-релятивистские параметры; $\beta=\gamma=1 ; v_{i}=\left|\dot{\mathbf{r}}_{i}\right| ; c-$ скорость света. $\left.\right|^{2}$

При создании точных эфемерид Луны, кроме гравитационных и релятивистских эффектов, необходимо учитывать влияние фигур Земли и Луны в математической модели. Ускорение Луны благодаря учету зональных и тессеральных гармоник в координатной системе $\xi \eta \zeta$ (см. рисунок) имеет вид [9]

$$
\left[\begin{array}{c}
\dot{\xi} \\
\ddot{\eta} \\
\ddot{\zeta}
\end{array}\right]=-\frac{\mu}{r^{2}}\left\{\sum_{n=1}^{n_{1}} J_{n}\left(\frac{a}{r}\right)^{n}\left[\begin{array}{c}
(n+1) P_{n}(\sin \varphi) \\
0 \\
-\cos \Theta P_{n}^{\prime}(\sin \varphi)
\end{array}\right]+\right.
$$

\footnotetext{
${ }^{2}$ Текст воспроизводится по работе автора [10].
} 


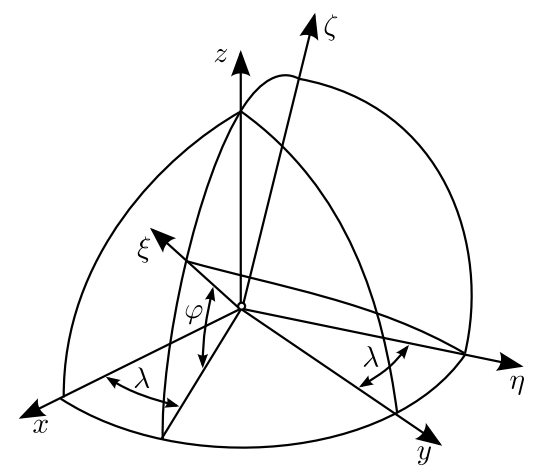

Система координат $\xi \eta \zeta$, для которой рассчитываются ускорения от несферического тела [The coordinate system $(\xi \eta \zeta)$ for which the calculated accelerations of a nonspherical body are presented]

$$
\left.+\sum_{n=1}^{n_{2}}\left(\frac{a}{r}\right)^{n} \sum_{m=1}^{n}\left[\begin{array}{c}
-(n+1) P_{n}^{m}(\sin \varphi)\left[C_{n m} \cos m \lambda+S_{n m} \sin m \lambda\right] \\
m \sec \varphi P_{n}^{m}(\sin \varphi)\left[-C_{n m} \sin m \lambda+S_{n m} \cos m \lambda\right] \\
\cos \varphi P_{n}^{\prime m}(\sin \varphi)\left[C_{n m} \cos m \lambda+S_{n m} \sin m \lambda\right]
\end{array}\right]\right\}
$$

где $\mu$-гравитационная постоянная; $r$-расстояние между центрами масс двух тел; $n_{1}$ и $n_{2}$ - максимальные степени зональных и тессеральных гармоник несферичных тел соответственно; $P_{n}(\sin \varphi)$ - полином Лежандра степени $n ; P_{n}^{m}(\sin \varphi)$ - присоединенный полином Лежандра степени $n$ и порядка $m$; $J_{n}$ - зональные гармоники от несферичности тела; $C_{n m}, S_{n m}$ - коэффициенты тессеральных гармоник; $\varphi$ - широта притягиваемого тела в фиксированной системе координат; $\lambda$ - восточная долгота притягиваемого тела. Вклад в инерциальное ускорение от несферичного тела возникает от взаимодействия его собственной фигуры с внешней точечной массой, представленной в координатной системе $\xi \eta \zeta$, где ось $\xi$ направлена вовне от несферичного тела к точечной массе, ось $\eta$ направлена на восток (лежит в селенографической плоскости $x y$, перпендикулярна оси $\xi)$ и, наконец, ось $\zeta$ направлена на север, образуя правую систему координат.

[Общая теория относительности, как и механика Ньютона, не свободна от недостатков. Важнейшим из них является вопрос о природе гравитации. В рамках общей теории относительности он, так же как и в теории гравитации Ньютона, рассматривается чисто феноменологически. Другим недостатком общей теории относительности является существенное усложнение дифференциальных уравнений в задаче $n$ тел. Решение этой задачи приходится искать в виде рядов по степеням малых параметров, при этом учитываются лишь линейные члены разложения и совершенно не исследуется вопрос о сходимости рядов. Основным недостатком как ньютоновской динамики, так и теории тяготения Эйнштейна является, на мой взгляд, наделение массы свойством, порождающим поле тяготения. В первом случае предполагается, что масса обладает свойством притяжения других материальных тел. Во втором случае масса наделяется свойством искривлять окружающее пространство. Все вышеперечисленные недостатки как ньютоновской динамики, так и общей теории относительности отражаются на степени точности и достоверности исследований, проводимых на их основе. $]^{3}$

\footnotetext{
${ }^{3}$ Текст воспроизводится по работе автора [5].
} 
Следует отметить, что в небесной механике Ньютона и Эйнштейна гравитационное поле Солнечной системы создается самими ее телами, однако это лишь рабочая гипотеза.

Возможна и другая точка зрения. Можно постулировать, что это гравитационное поле создано всей материей Вселенной. В этом случае силовое поле, созданное Вселенной, воздействует на тела, принуждая их к соединению.

Развитие идей об эфирной природе тяготения принадлежит современнику Ньютона Р. Гуку [6]. Согласно его представлению, колебание атомов материального тела передаются эфиру, распространяются в последнем и, достигая других тел, вызывают их притяжение к данному телу.

[Объяснения причины гравитации были высказаны Б. Риманом [11], а впоследствии А. Пуанкаре [12].

Рассуждая о природе тяготения, Б. Риман пишет [11, стр. 472]: «Существующую (...) в каждой точке пространства определенную по величине и направлению силу ускорения я пытаюсь объяснить движением некоей субстанции, наполняющей все бесконечное пространство. Эту субстанцию можно представить себе как физическое пространство, точки которого движутся в геометрическом пространстве.

На основании этого допущения все воздействия весомых тел на весомые тела передаются в пустом пространстве посредством названной субстанции. Таким образом, формы движения, лежащие в существе света и теплоты, посылаемых небесными телами, суть не что иное, как формы движения этой субстанции. Но названные явления, именно тяготение и распространение света сквозь пустое пространство, - единственные, которые должны были бы быть объяснены только движением этой субстанции».

А. Пуанкаре высказывает схожие с Б. Риманом суждения о причине тяготения. Говоря о тяготении, А. Пуанкаре пишет [12, стр. 503]: «В то время как в ньютоновской механике количество энергии движущегося тела зависит от инерции тела, находящегося в движении, здесь энергия зависит от того, что называют инерцией эфира по отношению к электромагнитным силам. Инерция эфира возрастает вместе со скоростью и становится бесконечно большой, когда скорость электрона приближается к скорости света. Таким образом, кажущаяся масса электрона возрастает со скоростью (...). При этом новом представлении постоянной массы материи не существует. Инерцией обладает не материя, а эфир; он один оказывает сопротивление движению, так что можно было бы сказать: нет материи, есть только дыры в эфире».

Таким образом, размышляя о природе тяготения, как Б. Риман, так и А. Пуанкаре приходят к тому, что причину тяготения следует искать не в наличии массы в материальном теле, а в свойстве бесконечного пространства, которое у Б. Римана называется движущейся субстанцией, а у А. Пуанкаре свойством эфира. $]^{4}$

Целью данной работы является создание математической модели, в которой первопричиной гравитации является взаимодействие движущейся материи с окружающей средой, а также решение полученных уравнений для исследования движения больших планет, Луны и Солнца и сопоставление полученных результатов с результатами других исследователей.

[После успехов кинетической теории газов В. Томсон писал [13]: «Хорошо

\footnotetext{
${ }^{4}$ Текст воспроизводится по работе автора [14].
} 
известная (.. ) кинетическая теория газов представляет собой столь важный шаг на пути к объяснению с помощью движения таких свойств тел, которые представляются нами статистическими, что едва ли можно удержаться от мысли, что в будущем появится полная теория материи, в которой все свойства последней будут рассматриваться лишь как атрибуты движения» ("Тhе British Association", Nature, 30, p. 417. doi: 10.1038/030410a0).

Для вывода дифференциальных уравнений движения допускается ряд упрощений: пространство, в котором движутся материальные тела, имеющие сферическую форму с равномерно распределенной плотностью, обладает свойством сжатия при изменении пространственной плотности. $]^{5}$ В любой заданный момент времени каждая фиксированная область пространства имеет свою определенную плотность. Очевидно, что в пространственных областях, где находятся материальные тела, плотность пространства выше по сравнению с окрестностью, окружающей это тело. При движении материальных тел происходит изменение состояния всей системы. В местах, где находились материальные тела, образуются пустоты, т.е. области, плотности которых значительно меньше плотности окружающей среды. Реакция пространства на образование пустот должна приводить к выравниванию плотностей или заполнению пустот окружающей средой. Таким образом, пространство сжимается, заполняя пустоты, образованные движущимися материальными телами. [Вывод дифференциальных уравнений движения основан на следующей идее. В каждый фиксированный момент времени материальное тело занимает в пространстве определенный объем. При перемещении тела пространство, занимаемое им в предыдущий момент времени, освобождается. При этом освободившееся пространство заполняется окружающей его средой, тем самым происходит сжатие окружающего пространства на величину объема, освобожденного движущимся объектом. $]^{6}$

В работах $[5,14,15]$ получены дифференциальные уравнения движения, основанные на новом принципе взаимодействия, которые имеют следующий вид:

$$
\begin{aligned}
\frac{d^{2} X}{d t^{2}} & =\sum_{i}\left(\frac{X_{i}-X}{\Delta_{i}}\right) \frac{3 a_{0 i} r_{0 i}^{2}}{\Delta_{i}^{2}+\Delta_{i} \sqrt[3]{\Delta_{i}^{3}-r_{0 i}^{3}}+\sqrt[3]{\left(\Delta_{i}^{3}-r_{0 i}^{3}\right)^{2}}} \\
\frac{d^{2} Y}{d t^{2}} & =\sum_{i}\left(\frac{Y_{i}-Y}{\Delta_{i}}\right) \frac{3 a_{0 i} r_{0 i}^{2}}{\Delta_{i}^{2}+\Delta_{i} \sqrt[3]{\Delta_{i}^{3}-r_{0 i}^{3}}+\sqrt[3]{\left(\Delta_{i}^{3}-r_{0 i}^{3}\right)^{2}}} \\
\frac{d^{2} Z}{d t^{2}} & =\sum_{i}\left(\frac{Z_{i}-Z}{\Delta_{i}}\right) \frac{3 a_{0 i} r_{0 i}^{2}}{\Delta_{i}^{2}+\Delta_{i} \sqrt[3]{\Delta_{i}^{3}-r_{0 i}^{3}}+\sqrt[3]{\left(\Delta_{i}^{3}-r_{0 i}^{3}\right)^{2}}}
\end{aligned}
$$

где $X, Y, Z$ - барицентрические координаты возмущаемого тела; $X_{i}, Y_{i}, Z_{i}-$ барицентрические координаты возмущающих тел;

$$
\Delta_{i}^{2}=\left(X_{i}-X\right)^{2}+\left(Y_{i}-Y\right)^{2}+\left(Z_{i}-Z\right)^{2}
$$

$r_{0 i}$ - эффективный радиус $i$-того тела; $a_{0 i}$ - соответствующее ускорение для $i$-того тела на расстоянии $r_{0 i}$ от центра массы.

\footnotetext{
${ }^{5}$ Текст воспроизводится по работе автора [5].

${ }^{6}$ Текст воспроизводится по работе автора [5].
} 
Сопоставим дифференциальные уравнения движения (1) и (4). Эти уравнения имеют как общие свойства, так и существенные различия. Как следует из уравнений (4), закон изменения ускорения отличен от закона обратной пропорциональности квадрату расстояния от произвольной точки до центра материального тела. Однако вследствие того, что размеры материальных тел существенно меньше расстояний между телами, отклонения ускорений для большинства материальных тел от закона обратной пропорциональности квадрату расстояний между ними оказывается незначительным.

Наиболее существенные различия дифференциальных уравнений (1) и (4) заключаются в предпосылках, на основе которых эти уравнения получены. В основе вывода уравнений (1) лежит закон всемирного тяготения и три аксиомы движения. Вывод уравнений (4) основан на более простых и естественных предположениях. В основе вывода уравнений (4) лежит принцип сжатия окружающего пространства относительно движущегося материального тела.

Сравним константы, входящие в уравнения (1) и (4). Постоянными в уравнениях (1) являются $k^{2} m_{i}$, где $k$-постоянная Гаусса, $m$-масса Земли и Луны, $m_{i}$ - массы возмущающих тел, $T$ - продолжительность сидерического года в средних солнечных сутках. Постоянными в уравнениях (4) являются $a_{0 i} r_{0 i}^{2}$, где $r_{0 i}$ - радиусы возмущающих тела, $a_{0 i}$ - ускорения на поверхности каждого возмущающего тела.

Таким образом, для нахождения констант, входящих в уравнение (1), требуется определить пять величин для каждого уравнения, значения которых не могут быть найдены путем прямых измерений. В тоже время для нахождения констант, входящих в уравнения (4), для каждого тела требуется найти две величины: $r_{0 i}$ - радиусы возмущающих тела, $a_{0 i}-$ ускорения на поверхности каждого возмущающего тела, значения которых могут быть найдены путем прямых измерений.

Следует отметить, что размерности постоянных, входящих в уравнения (1) и (4), совпадают. В системе СГС $k^{2} m_{i}$ и $a_{0 i} r_{0 i}^{2}$ имеют размерность см ${ }^{3} / \mathrm{ceK}^{2}$, т. е. изменение объема в единицу времени является постоянной величиной. Как раз этот принцип лежит в основе вывода уравнений (4).

[Дифференциальные уравнения (4) имеют более сложный вид по сравнению с уравнениями (1). Однако они значительно проще дифференциальных уравнений движения, учитывающих релятивистские эффекты [7,9].

Под эффективным радиусом материального тела понимается такой радиус сферы, которому соответствует сжатие пространства на величину фактического объема. Из физических соображений ясно, что тела различной плотности и одинакового размера не могут иметь равные эффективные радиусы. Их значения можно определить путем согласования решения уравнений (4) с наблюдениями.

Следует отметить, что система дифференциальных уравнений (4) не содержит масс тел и силовых взаимодействий. Дифференциальные уравнения (4) сохраняют принцип дальнодействия, так как в уравнениях (4) отсутствуют силовые взаимодействия между телами и процесс сжатия пространства относительно всех материальных тел происходит одновременно.

Таким образом, дифференциальные уравнения (1) и (4) описывают движение материальных тел в барицентрической системе координат. Уравнения (1) - обычные дифференциальные уравнения движения в ньютоновской фор- 
ме. Вывод дифференциальных уравнений (4) основан на новом принципе взаимодействия материальных тел, в которых отсутствует явно понятие массы и силы. Понятие ускорения определяется через закон изменения величины радиус-вектора при сжатии пространства в процессе движения материального тела. При $r_{0 i} \rightarrow 0$, т. е. при замене материальных тел конечных размеров материальными телами с бесконечно малыми линейными размерами, уравнения (4) обращаются по форме в уравнения (1). $]^{7}$

Следует напомнить, что в формулах (4) значения радиусов взаимодействующих тел сжимаются на величину, которая соответствует предельной плотности взаимодействующих тел. Радиусы Солнца и внешних планет при этом уменьшаются в 2.3 раза, а радиусы внутренних планет-в 2 раза. После сжатия небесные тела могут принять произвольную форму. В первом приближении можно считать, что тела, имеющие форму эллипсоида вращения, после сжатия примут сферическую форму. Тогда уравнения (4) будут справедливы для исследования движения всех небесных объектов, имеющих форму эллипсоида вращения, т. е. нет необходимости в использовании формул (3). В данном случае такими телами являются Солнце и все большие планеты.

В настоящее время разработан ряд высокоточных численных теорий движения больших планет [16-18]. Наиболее известной из них является численная теория, созданная сотрудниками NASA Ньюхалом, Стендиншем, Вильямсом $[9,19]$. Ими создан банк данных координат больших планет, Луны и Солнца DE405 на интервале времени с 2305424.5 J.D. (1599 Jan 5) по 2525008.5 J.D. (2201 Feb 20). Координаты планет в банке данных хранятся в форме коэффициентов полиномов Чебышева, которые обеспечивают достаточно плотную форму записи на диске. Координаты и скорости внутренних планет, полученные с помощью банка данных DE405, согласованы с радиолокационными наблюдениями, а координаты всех планет согласованы с оптическими наблюдениями.

С целью проверки эффективности математической модели, описывающей движение больших планет, Луны и Солнца системой дифференциальных уравнений (4), в настоящей работе проведены исследования движения этих объектов на интервале времени с 1602 по 2193 гг, результаты вычислений сопоставлены с банком данных DE405. Начальные данные координат $X, Y$, $Z$ и скоростей $\dot{X}, \dot{Y}, \dot{Z}$, фактических радиусов $r_{0 i}$ и ускорений $a_{0 i}$ приведены в табл. 1.8

Оценка точности численных и аналитических теорий движения небесных тел проверяется путем сравнения полученных координат объектов с наблюдениями. Погрешность в координатах планет, найденных по оптическим наблюдениям, составляет 1000-1500 км, что соответствует $6.7 \cdot 10^{-6}-1.0 \cdot 10^{-5}$ a.e. [18]. Точность радиолокационных наблюдений на два порядка выше, что составляет $6.7 \cdot 10^{-8}-1.0 \cdot 10^{-7}$ а. е. В связи с этим при сравнении координат и компонент скоростей на интервале времени с 1602 по 2193 гг. в значениях табл. 2-13 удерживалось 7 знаков после запятой.

Наряду с математической моделью, представленной в форме системы обыкновенных дифференциальных уравнений и описывающей движение небесных

\footnotetext{
${ }^{7}$ Текст воспроизводится по работе автора [14].

${ }^{8}$ Таблица воспроизводится по работе автора [14].
} 
Таблица 1

Начальные данные (координаты (а.е.), скорости (а.е./сут), радиус (км) и ускорение $\left(\right.$ км $\left.\left./ \mathrm{c}^{2}\right)\right)$ для планет, Луны и Солнца на момент времени $T=2440400.5$ J.D. (1969 06 28.0) [The initial data (coordinates (au), speeds (au/day), radius (km), and acceleration $\left.\left(\mathrm{km} / \mathrm{sec}^{2}\right)\right)$ for the planets, the Moon and the Sun on the time $T=2440400.5$ J.D. (1969 06 28.0)]

\begin{tabular}{|c|c|c|}
\hline \multicolumn{3}{|c|}{ Меркурий [Mercury] } \\
\hline$X=0.361762714604$ & $Y=-0.090781967730$ & $Z=-0.085714983182$ \\
\hline$\dot{X}=0.00336749391398$ & $\dot{Y}=0.02489452044680$ & $\dot{Z}=0.01294630068860$ \\
\hline$r=2.424 \cdot 10^{3}$ & $w=0.369066656 \cdot 10^{-2}$ & \\
\hline \multicolumn{3}{|c|}{ Венера |Venus| } \\
\hline$X=0.612751941342$ & $Y=-0.348365368495$ & $Z=-0.195278288980$ \\
\hline$\dot{X}=0.0109520683617$ & $\dot{Y}=0.0156176843653$ & $\dot{Z}=0.00633110555360$ \\
\hline$r=6.100 \cdot 10^{3}$ & $w=0.899027143 \cdot 10^{-2}$ & \\
\hline \multicolumn{3}{|c|}{ Земля [Earth] } \\
\hline$X=0.120527237123$ & $Y=-0.925814243017$ & $Z=-0.401527009924$ \\
\hline$\dot{X}=0.01680396477149$ & $\dot{Y}=0.00175034387379$ & $\dot{Z}=0.000759242499157$ \\
\hline$r=6.20315 \cdot 10^{3}$ & $w=0.979831333 \cdot 10^{-2}$ & \\
\hline \multicolumn{3}{|c|}{ Луна [Moon] } \\
\hline$X=0.119719059795$ & $Y=-0.927808873018$ & $Z=-0.402614272585$ \\
\hline$\dot{X}=0.01740504958809$ & $\dot{Y}=0.00158289841317$ & $\dot{Z}=0.00067368035418$ \\
\hline$r=1.738 \cdot 10^{3}$ & $w=0.163 \cdot 10^{-2}$ & \\
\hline \multicolumn{3}{|c|}{ Mapc [Mars] } \\
\hline$X=-0.110186074283$ & $Y=-1.327599456133$ & $Z=-0.605889132614$ \\
\hline$\dot{X}=0.0144816530597$ & $\dot{Y}=0.00024246311776$ & $\dot{Z}=-0.000281520734247$ \\
\hline$r=4.812 \cdot 10^{3}$ & $w=0.469169773 \cdot 10^{-2}$ & \\
\hline \multicolumn{3}{|c|}{ Юпитер [Jupiter] } \\
\hline$X=-5.379706898836$ & $Y=-0.830480581460$ & $Z=-0.224828700228$ \\
\hline$\dot{X}=0.00109201154301$ & $\dot{Y}=-0.00651811656579$ & $\dot{Z}=-0.00282078316536$ \\
\hline$r=7.144 \cdot 10^{4}$ & $w=2.484123914 \cdot 10^{-2}$ & \\
\hline \multicolumn{3}{|c|}{ Сатурн [Saturn] } \\
\hline$X=7.894392441979$ & $Y=4.596477801627$ & $Z=1.558697573530$ \\
\hline$\dot{X}=-0.00321755523930$ & $\dot{Y}=0.00433580985896$ & $\dot{Z}=0.00192864656566$ \\
\hline$r=6.044 \cdot 10^{4}$ & $w=1.038017148 \cdot 10^{-2}$ & \\
\hline \multicolumn{3}{|c|}{ Уран [Uranus] } \\
\hline$X=-18.265398306822$ & $Y=-1.161944505518$ & $Z=-0.250103483937$ \\
\hline$\dot{X}=0.000221188417418$ & $\dot{Y}=-0.00376247593285$ & $\dot{Z}=-0.00165101470307$ \\
\hline$r=2.486 \cdot 10^{4}$ & $w=0.939014231 \cdot 10^{-2}$ & \\
\hline \multicolumn{3}{|c|}{ Нептун [Neptune] } \\
\hline$X=-16.055042583768$ & $Y=-23.942181216179$ & $Z=-9.400156723549$ \\
\hline$\dot{X}=0.00264277104336$ & $\dot{Y}=-0.00149831445536$ & $\dot{Z}=-0.000679041903018$ \\
\hline$r=2.650 \cdot 10^{4}$ & $w=1.000508651 \cdot 10^{-2}$ & \\
\hline \multicolumn{3}{|c|}{ Плутон [Pluto] } \\
\hline$X=-30.483319603999$ & $Y=-0.872478355496$ & $Z=8.911563040990$ \\
\hline$\dot{X}=0.000322210447723$ & $\dot{Y}=-0.00314357030215$ & $\dot{Z}=-0.00107794882974$ \\
\hline$r=2.900 \cdot 10^{3}$ & $w=0.1167183115 \cdot 10^{-2}$ & \\
\hline \multicolumn{3}{|c|}{ Солнце [Sun] } \\
\hline$X=0.0045025081562339$ & $Y=0.0007670747009324$ & $Z=0.0002660568051770$ \\
\hline$\dot{X}=-0.3517482096 \cdot 10^{-6}$ & $\dot{Y}=0.51776253996 \cdot 10^{-5}$ & $\dot{Z}=0.22291018544 \cdot 10^{-5}$ \\
\hline$r=6.9596405726 \cdot 10^{5}$ & $w=273.9920358 \cdot 10^{-3}$ & \\
\hline
\end{tabular}


тел, важную роль в процессе решения играет численный метод интегрирования данной системы.

Численное интегрирование уравнений движения (4) было проведено модифицированным методом Эверхарта 27 порядка с шагом интегрирования 3 дня [20, 21].

Отметим различия математических моделей, на основании которых строились численные теории движения больших планет, Луны и Солнца в DE405 и в данной работе. В математической модели DE405 учитывались релятивистские эффекты, при этом наряду с планетными возмущениями учитывались возмущения на движение Марса и Юпитера от 400 самых крупных астероидов, движущихся в поясе астероидов [19]. Кроме этого, движение Луны и Земли рассматривалось с учетом их фигур, отличных от тел сфероидальной формы.

В математической модели, предлагаемой в данной работе, уравнения (4) являются нерелятивистскими, предполагается, что Земля и Луна имеют форму сфероида. В отличие от математической модели DE405, движение Марса и Юпитера рассматривается без учета влияния на их движение пояса астероидов.

Следует отметить, что математическая модель для исследования движений больших планет, Луны и Солнца, представленная в виде систем дифференциальных уравнений (4), значительно проще математической модели, используемой при создании банка данных DE405.

Важно знать, насколько результаты вычислений координат и компонент скоростей больших планет, Луны и Солнца, полученные с помощью описанных здесь математических моделей, согласуются друг с другом.

В табл. 2-13 представлены координаты и компоненты скоростей больших планет, Луны и Солнца на интервале времени с 1602 по 2193 гг. Данные координат и компонент скоростей приведены на 0 часов гринвичского времени соответствующей даты, при этом в первой строке указаны данные банка DE405, во второй строке - координаты и компоненты скоростей, полученные путем решения уравнений (4).

Результаты сопоставления численного интегрирования уравнений движения (4) с данными банка данных DE405 приведены в табл. 2-13 и 14-17.

В первом столбце табл. 2 приведено время в календарной и юлианской дате на стандартные моменты, в остальных столбцах - координаты и скорости Солнца по DE405 и вычисленные по формулам (8). Как видно из табл. 2, координаты и компоненты скоростей Солнца, вычисленные двумя различными методами, отличаются друг от друга в шестом и седьмом знаках после запятой соответственно, что находится в пределах погрешностей наблюдений.

В табл. 3 и 14 приведены координаты и компоненты скоростей и элементы орбит Меркурия. Как показывают результаты вычислений, координаты, компоненты скоростей и элементы орбит, найденные путем решения уравнений (4) и с помощью DE405, полностью согласуются друг с другом, при этом расхождение координат и компонент скоростей находятся в пределах как оптических, так и радиолокационных погрешностей наблюдений.

В табл. 4 и 15 представлены координаты, компоненты скоростей и элементы орбит Венеры. Сравнение элементов орбит (см. табл. 15) показывает, что кроме аргумента перигелия, остальные элементы орбит, полученные на 
물

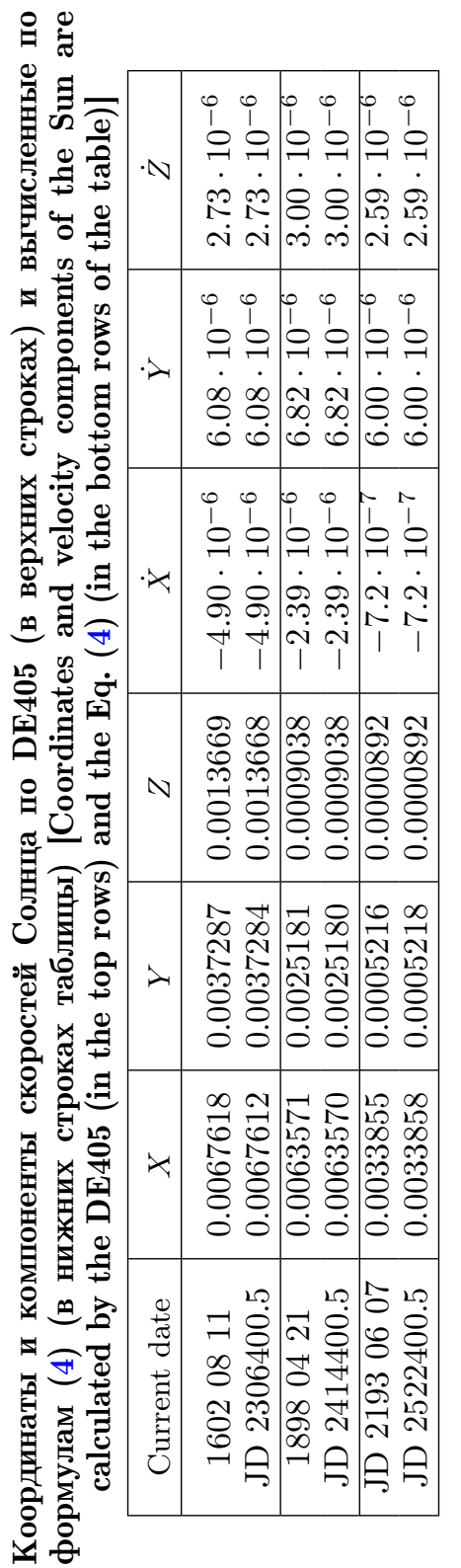

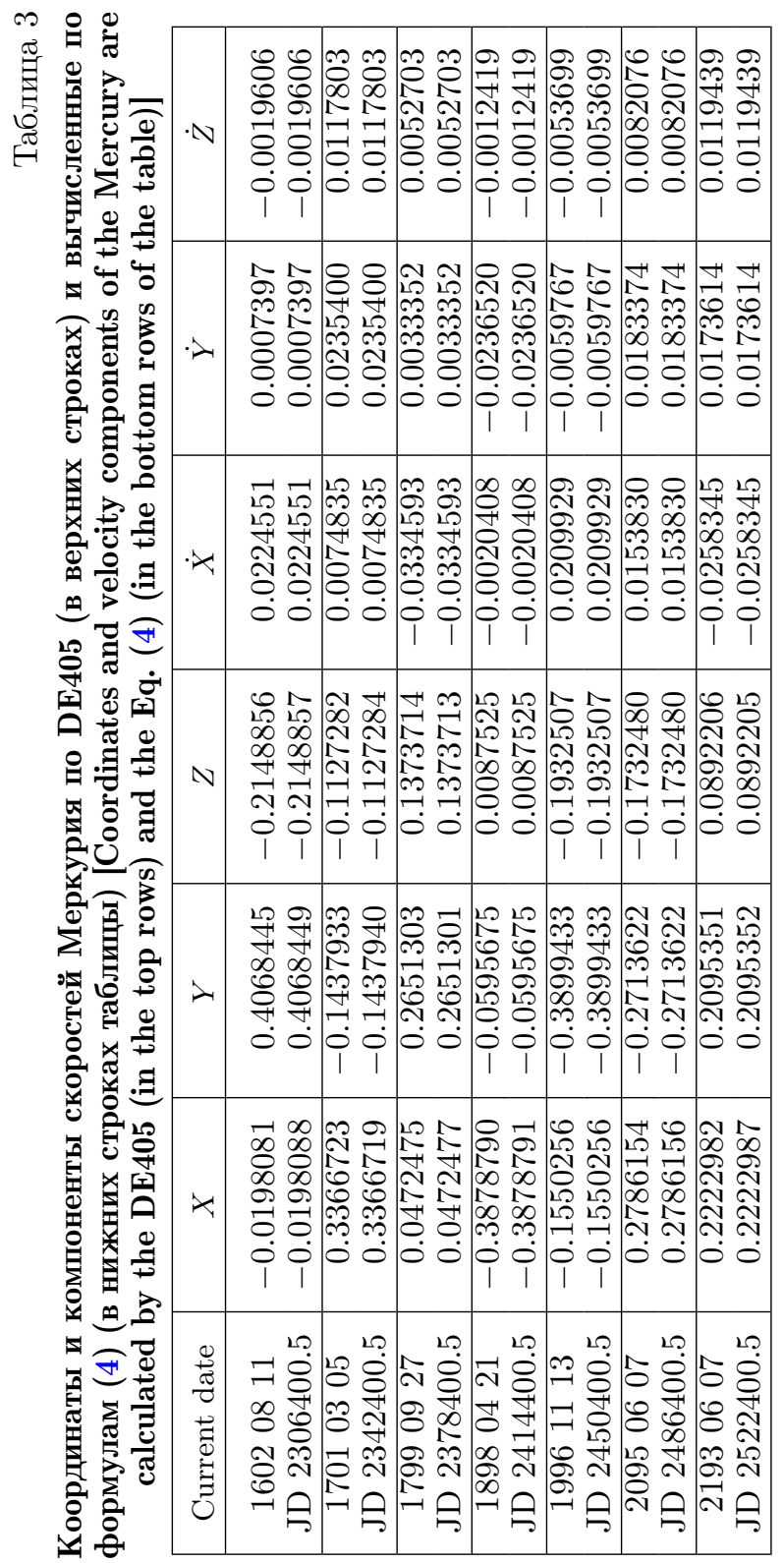



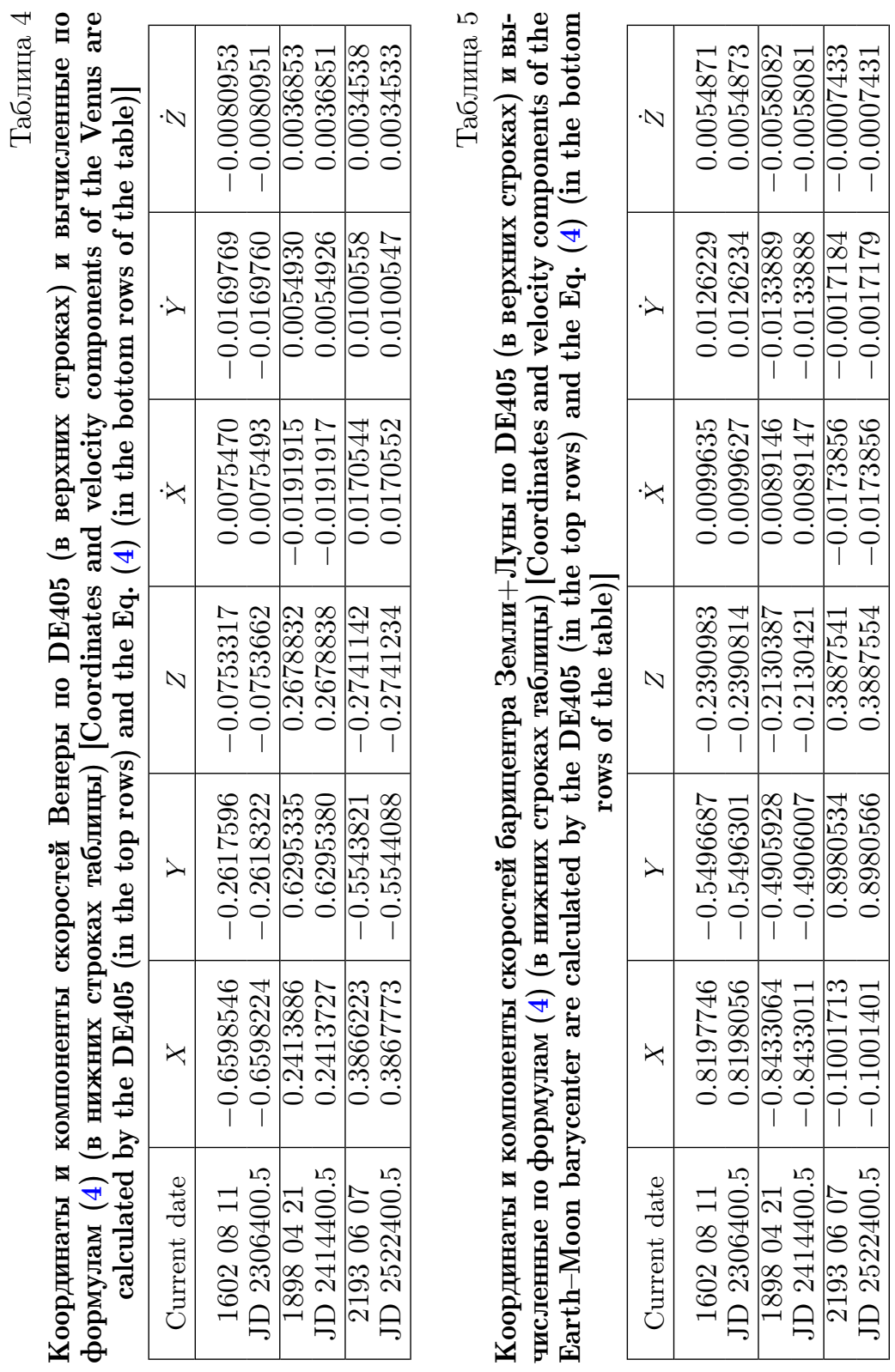


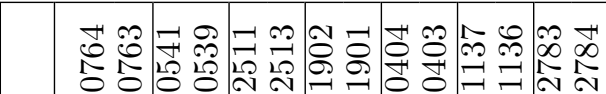

- N

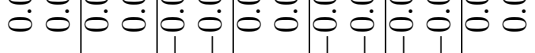

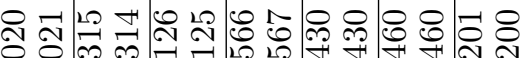
ต

- $\lambda$ 용

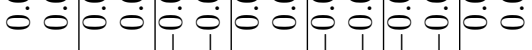

栗

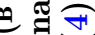

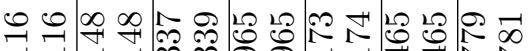

든든ㄷำ

- $x$ ¿

○

舟

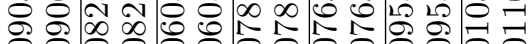

N ○

ช

a

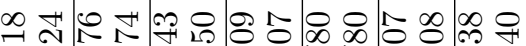

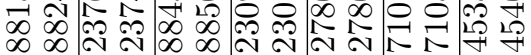

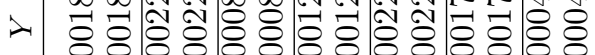

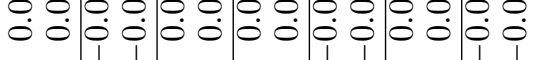

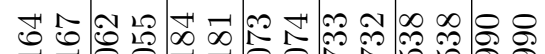
ت

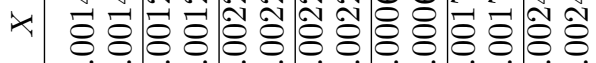

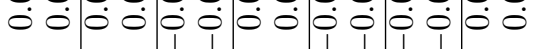

宩

造

है

을 울

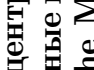

要要

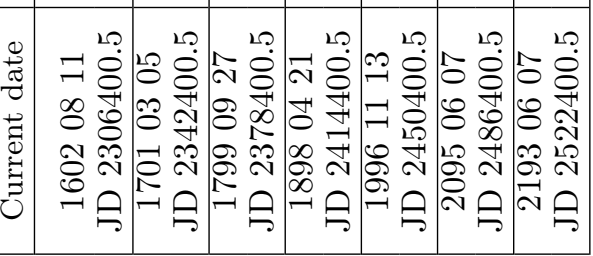

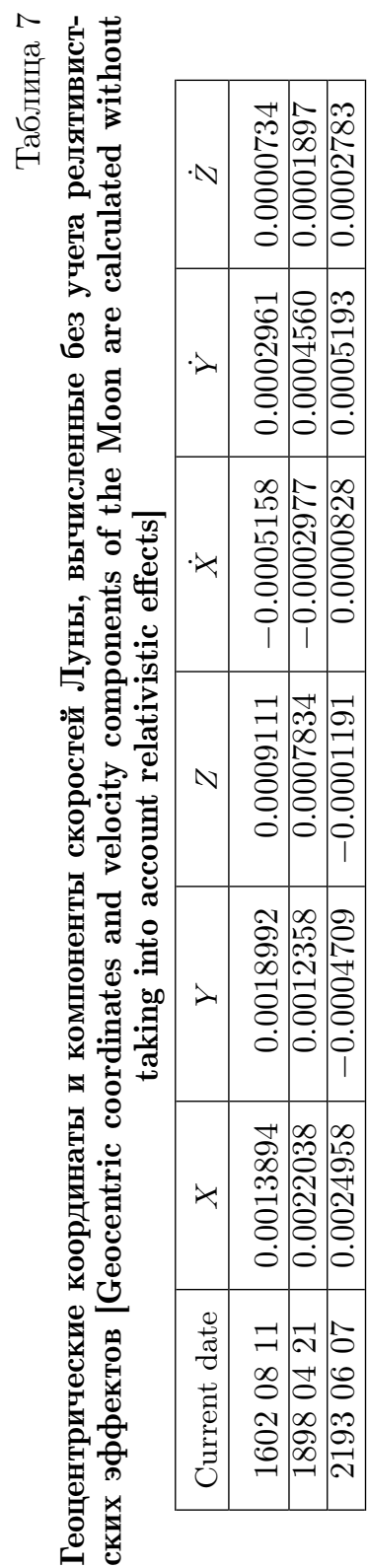



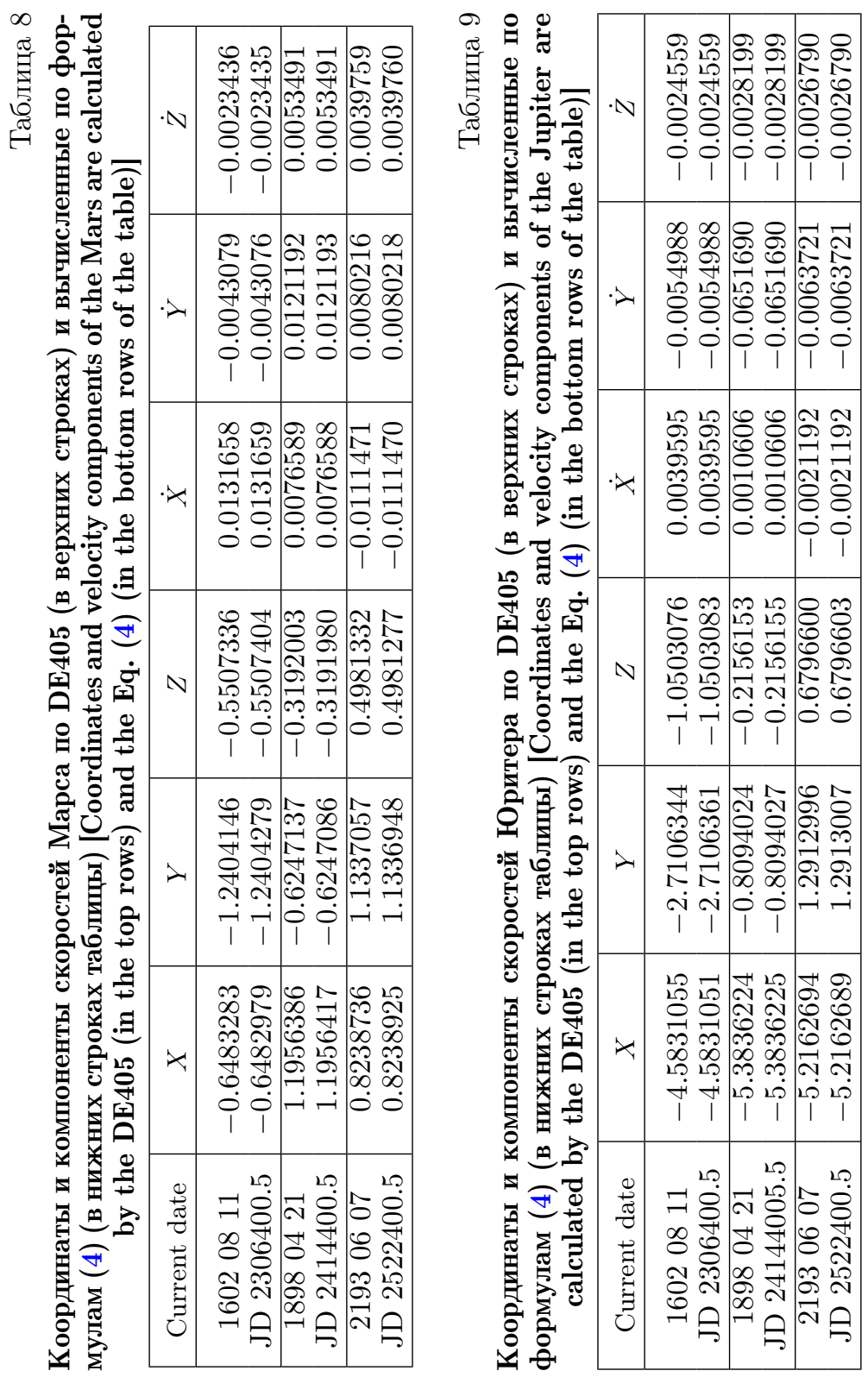
을

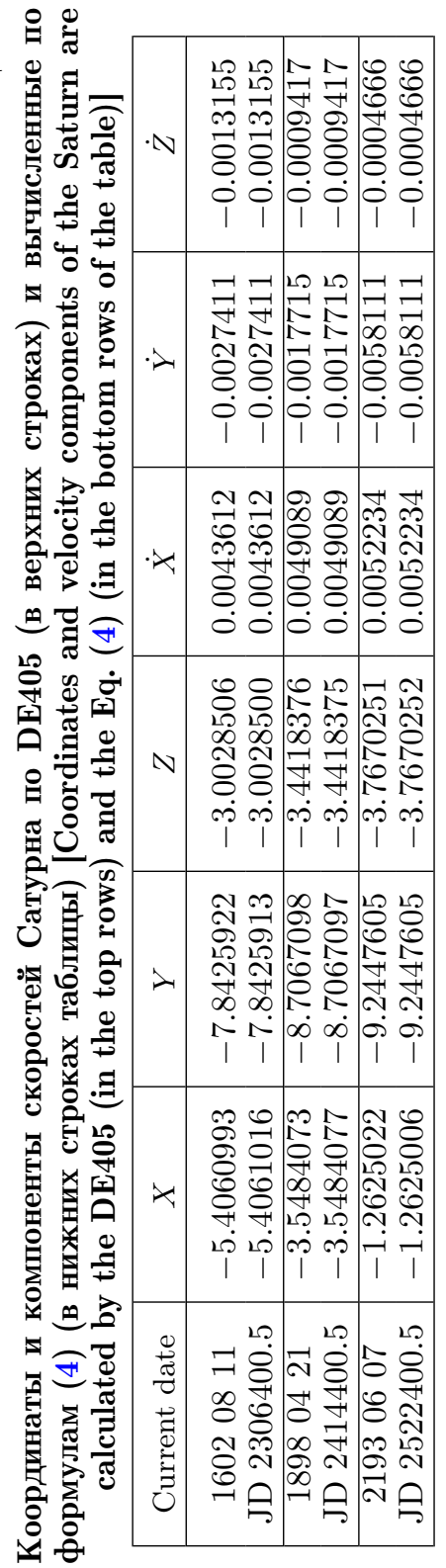

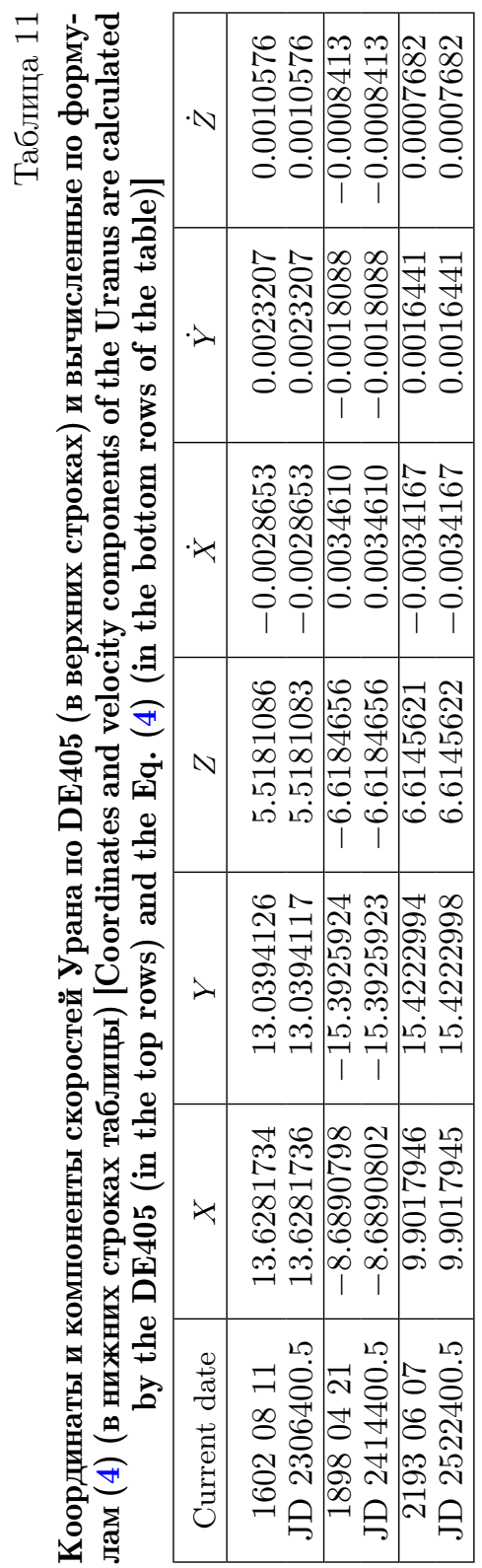


ป

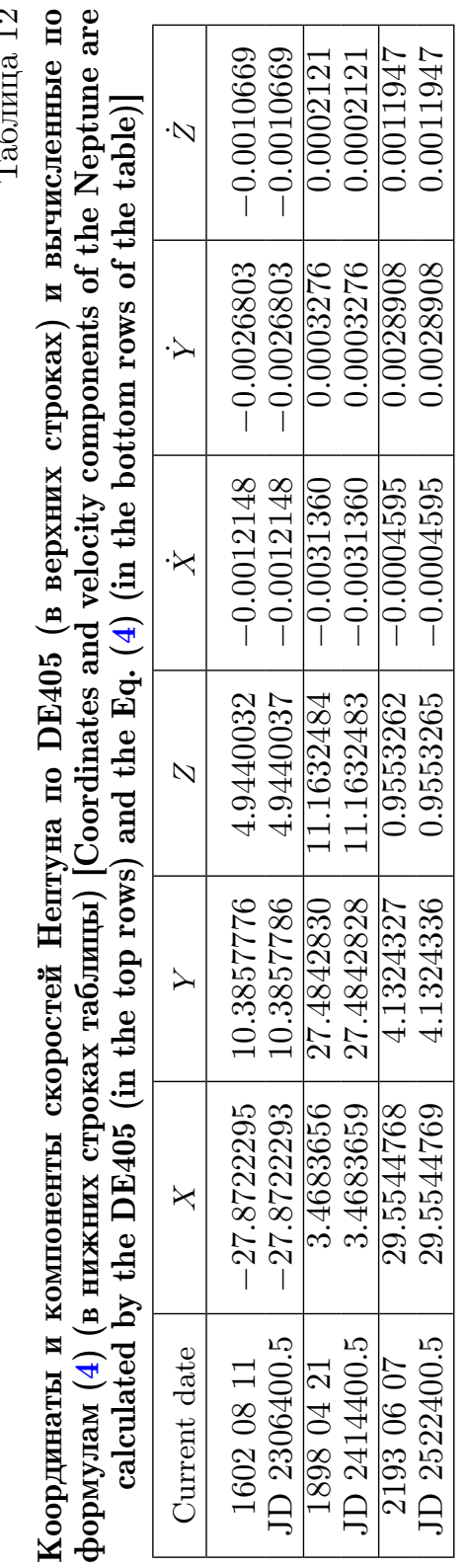

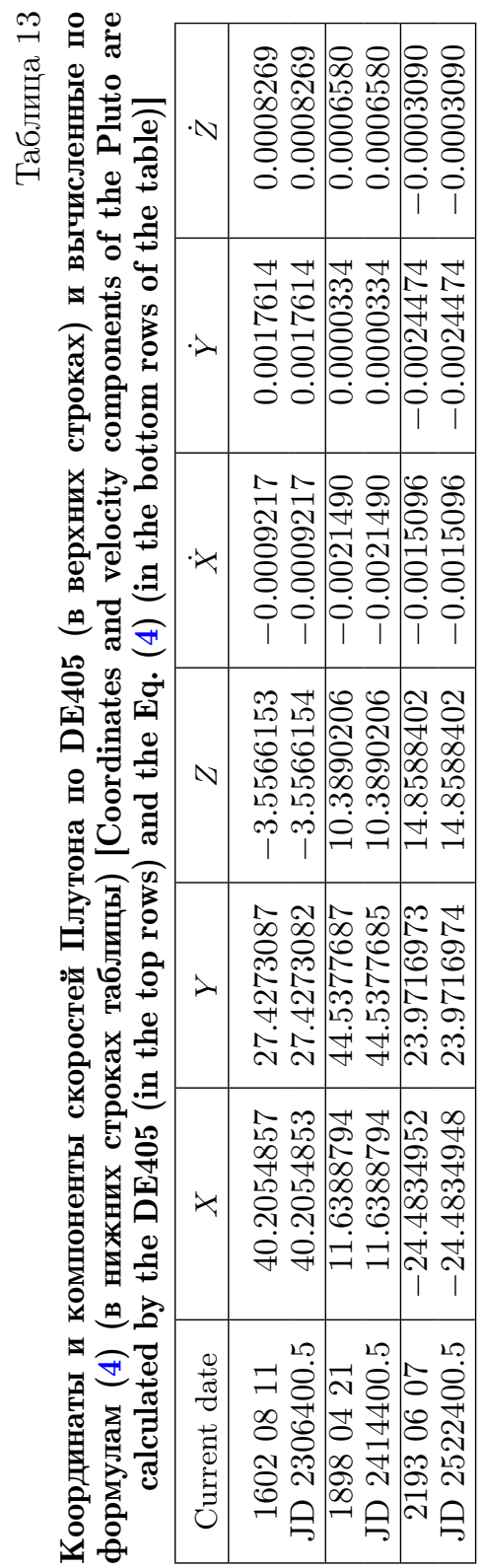




\begin{tabular}{|c|c|c|c|c|c|c|c|c|}
\hline 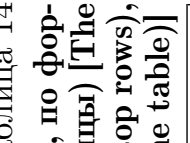 & $\sim$ & 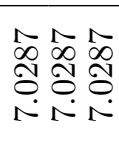 & 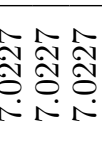 & $\begin{array}{lll}0 & 0 & 0 \\
0 & 0 & 0 \\
0 & 0 & 0 \\
0 & 0 & 0 \\
N & N & N\end{array}$ & 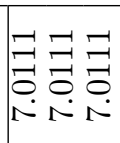 & 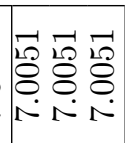 & 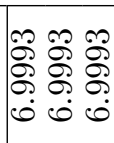 & 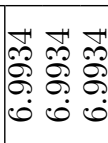 \\
\hline 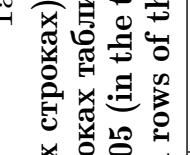 & $\sigma$ & 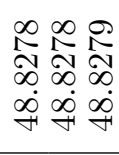 & 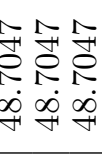 & $\mid \begin{array}{lll}\infty & \infty & \infty \\
\infty & \infty & \infty \\
\infty & \infty & \infty \\
2 & 1 & 1 \\
\infty & \infty & \infty \\
\sim & \infty & \infty \\
\sim & \infty & \neq\end{array}$ & 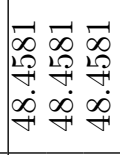 & 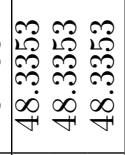 & 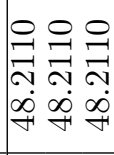 & 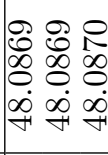 \\
\hline 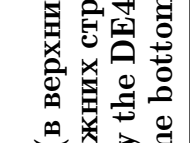 & 3 & 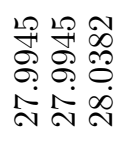 & 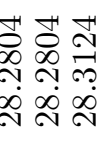 & 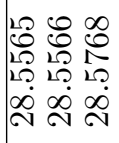 & 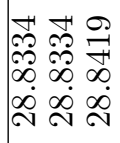 & 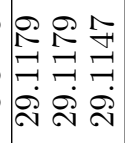 & 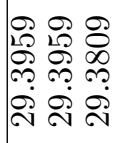 & 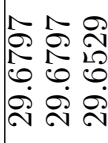 \\
\hline 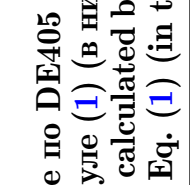 & 0 & 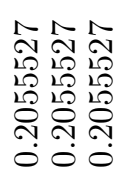 & 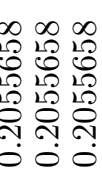 & 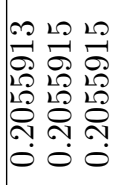 & 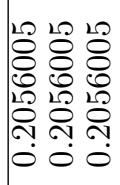 & 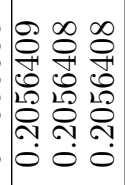 & 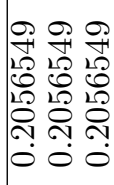 & $\begin{array}{lll}1 & 0 & 10 \\
1 & 0 & 1 \\
0 & 0 & 0 \\
0 & 0 & 0 \\
0 & 0 & 0 \\
0 & 0 & 0 \\
0 & 0 & 0 \\
& 0 & 0 \\
0 & 0 & 0\end{array}$ \\
\hline 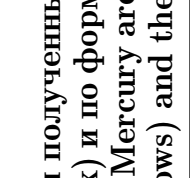 & $\theta$ & 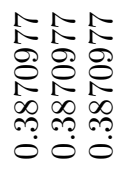 & 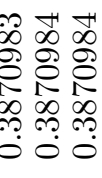 & 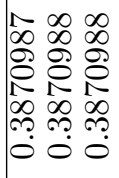 & 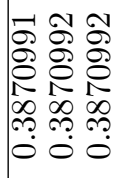 & 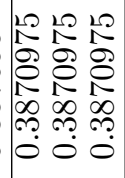 & 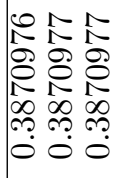 & 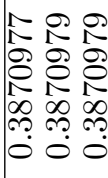 \\
\hline 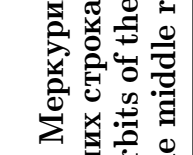 & $z$ & 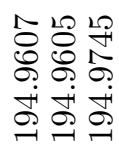 & 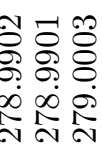 & 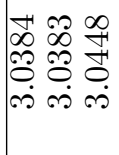 & 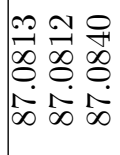 & 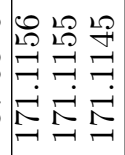 & 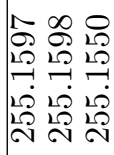 & 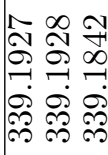 \\
\hline 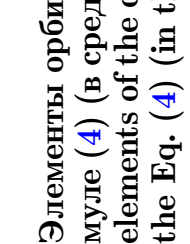 & 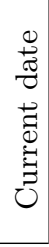 & 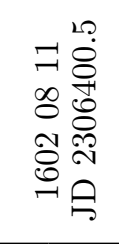 & 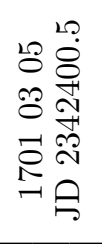 & 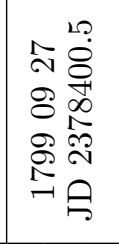 & 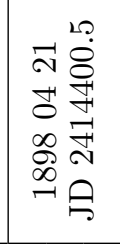 & 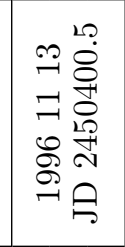 & 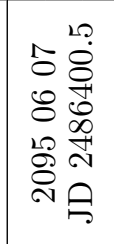 & 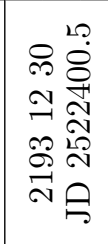 \\
\hline
\end{tabular}




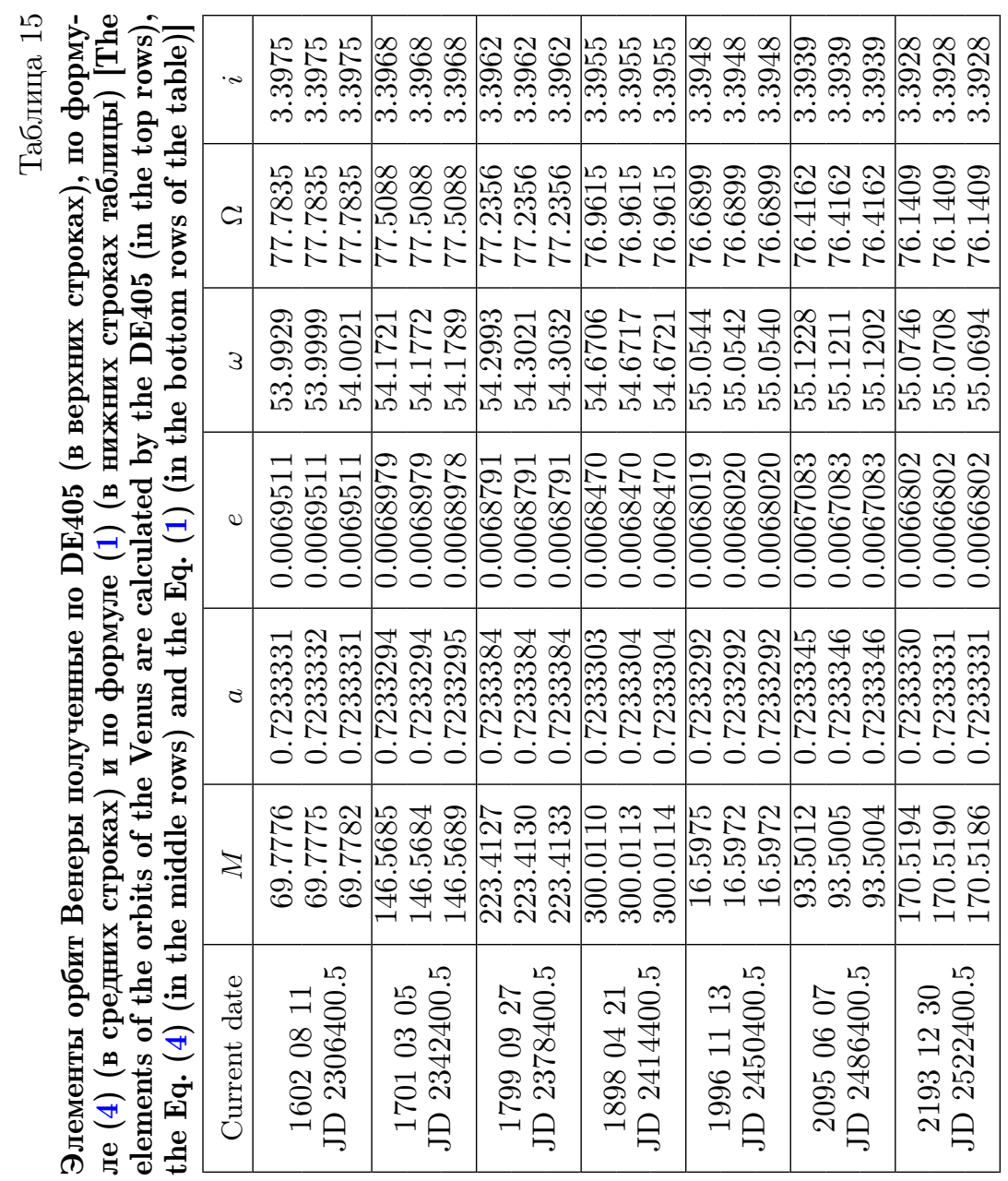




\begin{tabular}{|c|c|c|c|c|c|c|c|c|}
\hline 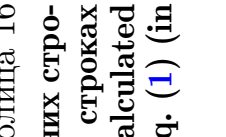 & $\sim$ & 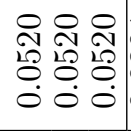 & 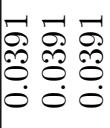 & 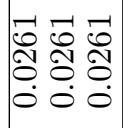 & 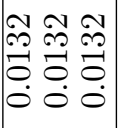 & 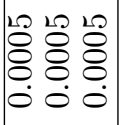 & $\begin{array}{l}\text { H } \\
\text { th }\end{array}$ & $\stackrel{0}{0}$ \\
\hline 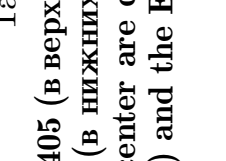 & $a$ & 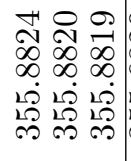 & 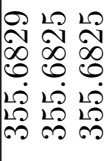 & 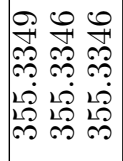 & 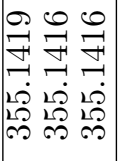 & 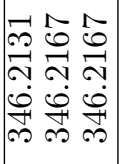 & 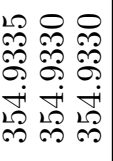 & 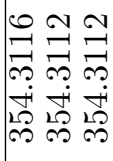 \\
\hline 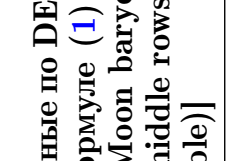 & 3 & 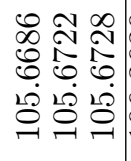 & $\begin{array}{lll} & 1 & 0 \\
0 & 0 & 0 \\
0 & 0 & 0 \\
0 & 0 \\
0 & 0 & 0 \\
0 & 0 & 8\end{array}$ & 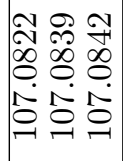 & 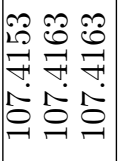 & 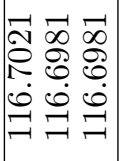 & & 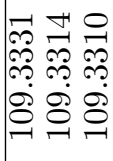 \\
\hline 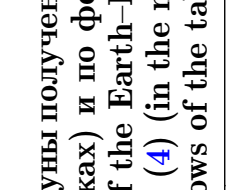 & 0 & 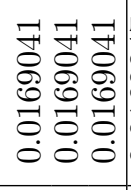 & 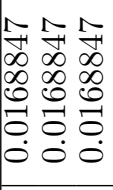 & $\left|\begin{array}{ccc}2 & 1 & 2 \\
0 & 0 & 0 \\
N & N & N \\
1 & N & 1 \\
0 & 0 & 0 \\
0 & 0 & 0 \\
0 & 0 & 0 \\
0 & 0 & 0\end{array}\right|$ & 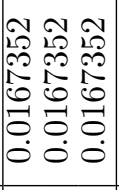 & 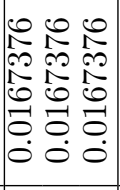 & $\begin{array}{ll}0 & 1 \\
0 & 1 \\
0 & 1 \\
0 & 1 \\
0 & 0 \\
0 & 0 \\
0 & 1\end{array}$ & $\begin{array}{lll}12 & 12 & 10 \\
2 & 2 & 2 \\
0 & 0 & 0 \\
10 & 10 & 10 \\
0 & 0 & 0 \\
0 & 0 & 0 \\
0 & 0 & 0 \\
0 & 0 & 0\end{array}$ \\
\hline 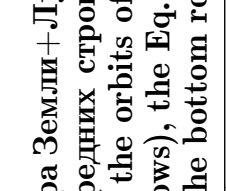 & $\nabla$ & 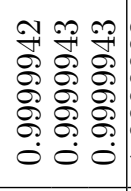 & 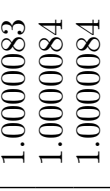 & 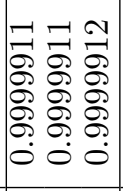 & 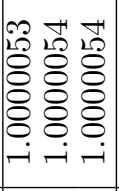 & 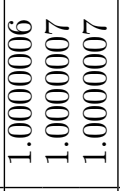 & 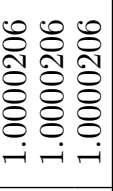 & 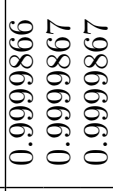 \\
\hline 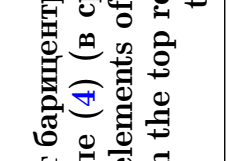 & $z$ & 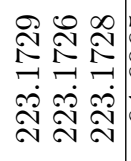 & 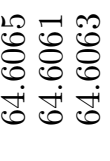 & 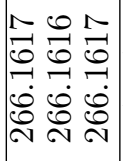 & 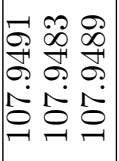 & 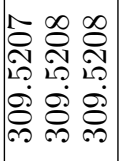 & 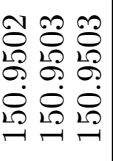 & 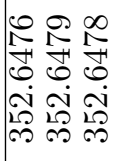 \\
\hline 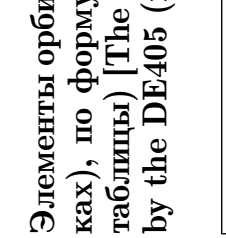 & 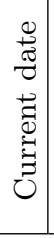 & 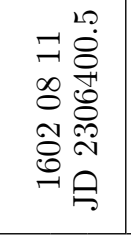 & 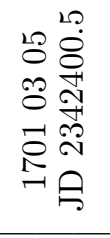 & 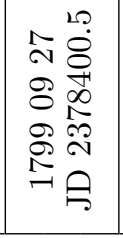 & 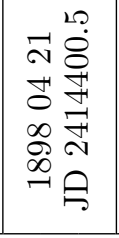 & 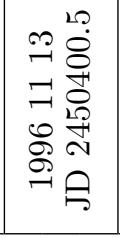 & 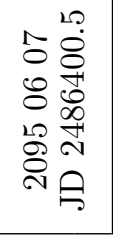 & 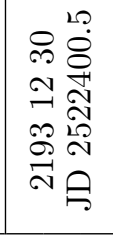 \\
\hline
\end{tabular}




\begin{tabular}{|c|c|c|c|c|c|c|c|c|}
\hline 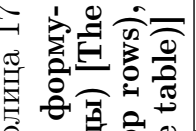 & $\sim$ & $\begin{array}{ll}\infty & \infty \\
\infty & \infty \\
\infty & \infty \\
\infty & \infty \\
- & \infty \\
- & -1\end{array}$ & 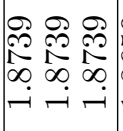 & $\left|\begin{array}{ccc}0 & 0 & 0 \\
2 & 10 & 1 \\
0 & 0 & 0 \\
\infty & \infty & \infty \\
-1 & -1 & -1\end{array}\right|$ & \begin{tabular}{lll}
$\not$ & $\overrightarrow{ }$ & \multicolumn{1}{c}{} \\
$\infty$ & $\infty$ & $\infty$ \\
2 & 20 & 2 \\
$\infty$ & $\infty$ & $\infty$ \\
- & -1 & -
\end{tabular} \mid & 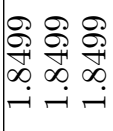 & 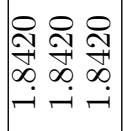 & $\begin{array}{lll}\infty & \infty & \infty \\
\cdots & \cdots & \cdots \\
2 & \cdots & \cdots \\
\infty & \infty & \infty \\
-1 & -1 & -1\end{array}$ \\
\hline 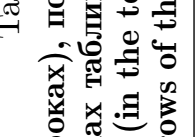 & $\sigma$ & 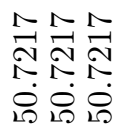 & 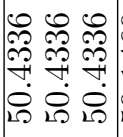 & 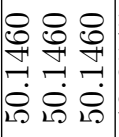 & 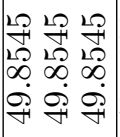 & 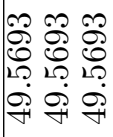 & 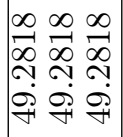 & 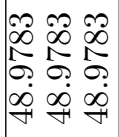 \\
\hline 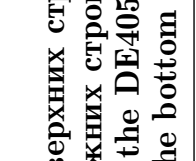 & 3 & 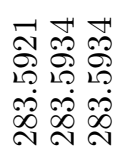 & 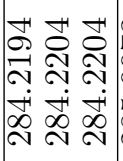 & 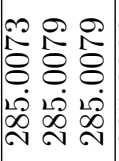 & 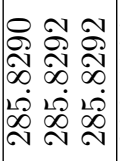 & 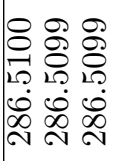 & 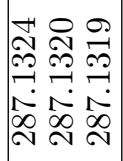 & 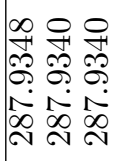 \\
\hline 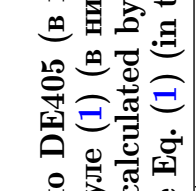 & 0 & 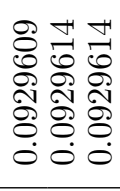 & 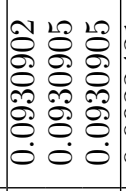 & 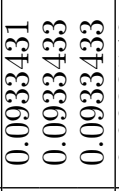 & 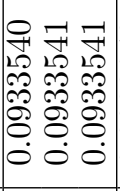 & 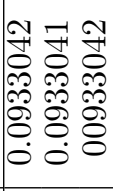 & 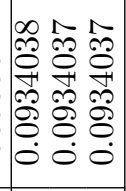 & \begin{tabular}{lll} 
& $\infty$ & $\infty$ \\
$\infty$ & 1 & 0 \\
0 & 0 & 0 \\
0 & 0 & 0 \\
\hdashline & 0 & 0 \\
0 & 0 & 0 \\
0 & 0 & 0 \\
0 & 0 & 0 \\
0 & 0 & 0
\end{tabular} \\
\hline 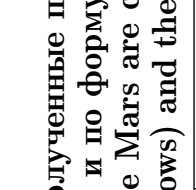 & 0 & 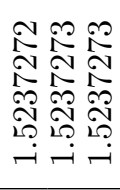 & 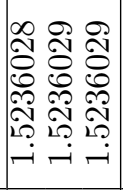 & 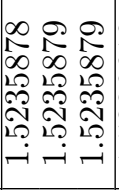 & 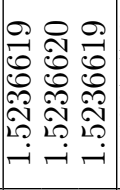 & 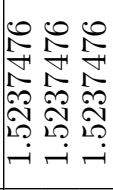 & 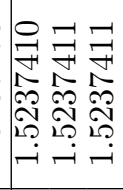 & 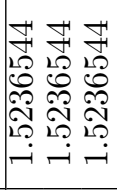 \\
\hline 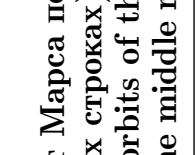 & $\Sigma$ & 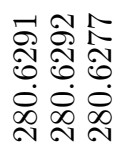 & 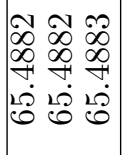 & 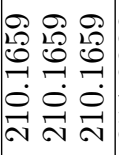 & 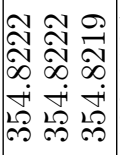 & 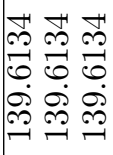 & 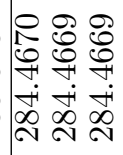 & 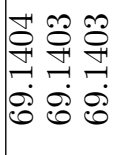 \\
\hline 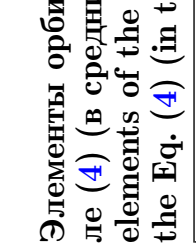 & 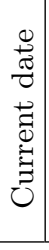 & 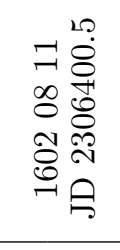 & 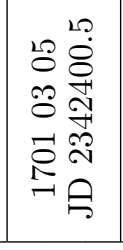 & 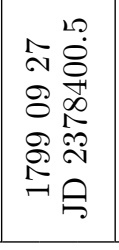 & 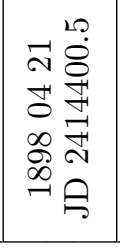 & 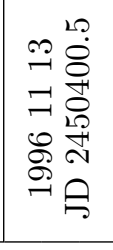 & 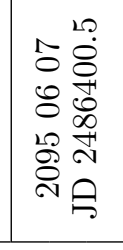 & 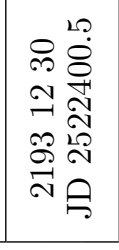 \\
\hline
\end{tabular}


основе решения уравнений (8) и найденные с помощью банка данных DE405, на одни и те же моменты времени практически совпадают. Отличие в вековом смещении аргумента перигелия (см. табл. 13) влечет различие координат и компонент скоростей, приведенных в табл. 4, при этом координаты отличаются между собой в пятом знаке после запятой, а компоненты скоростей в шестом знаке. Средние аномалии, найденные двумя методами, различаются между собой в пределах погрешности наблюдений. Вследствие того, что средняя аномалия определяет положение небесного тела на орбите, а величина аргумента перигелия не может быть получена непосредственно из наблюдений, из-за малого различия вековых смещений перигелия Венеры (см. табл. 13), не представляется возможным оценить его истинное вековое смещение.

В табл. 5 и 16 приведены координаты, компоненты скоростей и элементы орбит барицентра Земли+Луны. Из сравнения элементов орбит, найденных двумя методами (см. табл. 16), следует, что отличие значений аргументов перигелиев для барицентра Земли+Луны на одни и те же моменты времени имеет место вследствие различия векового смещения аргумента перигелия, найденного с помощью решения уравнений (4) и по DE405. Из-за малого различия вековых изменений аргумента перигелия Земли-Луны, полученных по DE405 и путем решения уравнений (4), не удается оценить его истинное смещение.

Отличие векового движения аргумента перигелия, найденного двумя методами, характерно и для Марса (см. табл. 8 и 17). Однако вследствие несущественных различий средних аномалий на всем интервале интегрирования установить истинную скорость движения аргумента перигелия Марса на основании данных наблюдений не представляется возможным.

В табл. 9-13 представлены координаты и компоненты скоростей внешних планет (Юпитер-Нептун) и Плутона на три стандартные даты - 11 августа 1602 г., 21 апреля 1898 г. и 7 июня 2193 г. Как видно из данных, приведенных в табл. 9-13, результаты координат и компонент скоростей, найденные двумя различными методами, практически не отличаются друг от друга.

Геоцентрические координаты и компоненты скоростей Луны приведены в табл. 6. Максимальное расхождение двух методов для различных геоцентрических координат Луны имеет место в разное время. При этом максимальное различие в координате $X$, как следует из табл. 6, имело место в 1701 г. 0.0000007 a.e., в координате $Y$ в 1799 г. - 0.0000007 a.е. и в координате $Z$ в 1602 г. -0.0000021 а.е., что соответствует $\Delta X=105 \mathrm{kм}, \Delta Y=105$ м и $\Delta Z=$ $=314$ м. Максимальное расхождение компонент скоростей 0.0000002 а.е./сут. Полученные расхождения в координатах и компонентах скоростей не являются существенными, т. к. находятся в пределах погрешностей оптических наблюдений.

В табл. 7 приведены геоцентрические координаты и скорости Луны, вычисленные без учета фигуры Земли и релятивистских эффектов, т. е. получены путем решения ньютоновских дифференциальных уравнений движения. Геоцентрические координаты и скорости Луны приведены для трёх моментов времени: 11 августа 1602 г., 21 апреля 1898 г. и на 7 июня 2193 г. Сопоставление данных, приведенных в табл. 6 и 7, показывает, что наибольшие расхождения координат и компонент скоростей имели место 11 августа 1602 г. и со- 
ставляли $\Delta X=0.000027$ a.e., $\Delta X=-0.0000174$ a.e. и $\Delta Z=-0.0000023$ a.e., что соответствует $\Delta X=-4039 \mathrm{kм}, \Delta Y=-2603$ км и $\Delta Z=-344$ км.

Из проведенного сопоставления следует, что координаты Луны, полученные на основе банка данных DE405 и путем решения ньютоновских дифференциальных уравнений движения, отличаются друг от друга более чем на порядок по сравнению с аналогичными сравнениями координат, полученных с помощью DE405 и путем решения уравнений (1). Полученные расхождения координат являются существенными, так как не находятся в пределах точности оптических наблюдений, что указывает на ограниченную возможность ньютоновских уравнений для исследования движения Луны на интервале времени порядка нескольких столетий.

В табл. 14-17 приведены элементы орбит внутренних планет, полученные тремя различными методами. В первой строке на каждую стандартную дату представлены элементы орбит, найденные с помощью банка данных DE405, во второй строке - на основании решения уравнений (4) и в третьей строке находятся элементы орбит, вычисленные с помощью решения ньютоновских уравнений (1). Это позволяет определить дополнительное вековое смещение аргумента перигелия по сравнению с ньютоновской моделью движения для каждой из планет.

Из сопоставления элементов орбит Венеры, барицентра Земли+Луны и Марса на различные моменты времени (см. табл. 15-17) следует, что различия элементов орбит, за исключением аргумента перигелия, полученные с помощью банка данных DE405 и на основе решения дифференциальных уравнений (4), на всем интервале интегрирования практически совпадают. На основании сопоставления аргументов перигелиев, найденных с помощью решения уравнений (4) и на основании решения ньютоновских уравнений, получены следующие дополнительные вековые смещения: у Меркурия оно составляет 42.91 секунд за столетие, у Венеры-2".19, у барицентра Земли+Луны - $0^{\prime \prime} .61$ и у Марса - $0^{\prime \prime} .08$.

Отсюда следует, что математическая модель движения Меркурия, основанная на использовании формул (4), полностью объясняет дополнительное вековое смещение долготы перигелия по сравнению с ньютоновской моделью движения небесных тел, равное 42.91 секунды за столетие.

В отличие от Меркурия, подобного согласования в смещении аргументов перигелиев у Венеры, Земли+Луны и Марса, полученных с помощью DE405 и уравнений (4), не наблюдается (см. табл. 14). У Венеры оно равно 2.19 секунды вместо 8".77, предсказанных релятивистской динамикой [7], у Земли十Луны $0^{\prime \prime} .61$ вместо $3^{\prime \prime} .83$ и у Марса - $0^{\prime \prime} .08$ секунды вместо $1^{\prime \prime} .28$. Следует отметить, что дополнительные смещения долгот перигелиев этих планет вычисляются с большими ошибками, значения которых по абсолютной величине составляют: для Венеры $- \pm 5^{\prime \prime} .28$, для барицентра Земли $+Л у н ы ~-~ \pm 1^{\prime \prime} .79$ и для Марса - $\pm 0^{\prime \prime} .27$ [7]. Вследствие этого не представляется возможным выявить истинные вековые смещения долгот перигелиев этих планет, основываясь на оптических наблюдениях.

Таким образом, в данной работе проведено сопоставление координат компонент и элементов орбит больших планет, Луны и Солнца, найденных тремя методами: на основе банка данных DE405, путем решения ньютоновских уравнений и с помощью решения уравнений (4). На основании проведенных 
исследований можно сделать следующие выводы.

Применение механики Ньютона для исследования движения не позволяет получить дополнительное смещение аргументов перигелия у внутренних планет. Кроме того, для построения теории движения Луны необходимо учитывать фигуру Земли, учет которой описывается отдельной системой дифференциальных уравнений.

Использование релятивистских уравнений больших планет, Луны и Солнца также не позволяет с помощью одной системы дифференциальных уравнений удовлетворительно согласовать движение этих тел с наблюдениями. Для движения Луны решается дополнительная система дифференциальных уравнений, учитывающая отклонения фигуры Земли от сфероида.

Проведенные исследования показали, что численные расчеты координат больших планет Луны и Солнца, основанные на решении системы дифференциальных уравнений (4), вполне удовлетворительно согласуются с координатами этих объектов, вычисленными с помощью банка данных DE405. Уравнения (4) не содержат членов, учитывающих несферичность Земли и Луны, являясь при этом нерелятивистскими уравнениями.

\section{ORCID}

Анатолий Федорович Заусаев: http://orcid.org/0000-0002-5035-9615

\section{БИБЛИОГРАФИЧЕСКИЙ СПИСОК}

1. Чеботарев Г. А. Аналитические и численные методъ небесной механики. М., Л.: Наука, 1965. 368 с.

2. Субботин М. Ф. Введение в теоретическую астрономию. М.: Наука, 1968. 800 с.

3. Le Verrier U. J. Theorie du movement de Mercure/ Annales de l'Observatoire imperial de Paris. vol. 5. Paris: Mallet-Bachelier, 1859. 195 pp.

4. Roseveare N. T. Mercury's Perihelion, from Le Verrier to Einstein. Oxford: Clarendon, 1982. 208 pp.

5. Заусаев А. Ф. Теория движения $n$ материальных тел, основанная на новом принципе взаимодействия // Вестн. Сам. гос. техн. ун-та. Сер. Физ.-мат. науки, 2006. № 43. C. 132-139. doi : 10.14498/vsgtu463.

6. Богородский А. Ф. Всемирное тяготение. Киев: Наукова думка, 1971. 352 с.

7. Брумберг В. А. Релятивистская небесная механика. М.: Наука, 1972. 384 с.

8. Рашевский П. К. Риманова геометрия и тензорный анализ. М.: Наука, 1967. 644 с.

9. Newhall X. X., Standish E. M., Williams J. G. DE 102: A numerically integrated ephemeris of the moon and planets spanning forty-four centuries // Astronomy and Astrophysics, 1983. vol. 125, no. 1. pp. 150-167.

10. Заусаев А. Ф. Исследование орбитальной эволюции 10 короткопериодических комет путем решения дифференциальных уравнений движения, полученных на основе нового принципа взаимодействия // Вестн. Сам. гос. техн. ун-та. Сер. Физ.мат. науки, 2007. № 1(14). C. 79-84. doi: 10.14498/vsgtu491.

11. Риман Б. Натурфилософия / Сочинения. М., Л.: ОГИЗ, ГИТТЛ, 1948. С. 467-477.

12. Пуанкаре А. Последние мысли / О науке. М.: Наука, 1983. С. 407-520.

13. Визгин В. П. Релятивистская теория тяготения (истоки и формирование, 1900-1915). М.: Наука, 1981. 352 с.

14. Заусаев А. Ф. Исследование движения планет, Луны и Солнца, основанное на новом принципе взаимодействия // Вестн. Сам. гос. техн. ун-та. Сер. Физ.-мат. науки, 2014. № 3(36). C. 118-131. doi: 10.14498/vsgtu1304.

15. Заусаев А. Ф., Заусаев А. А. Математическое моделирование орбиталъной эволючии малых тел Солнечной системы. М.: Машиностроение, 2008. 250 с. 
16. Питьева Е. В. Современные численные теории движения Солнца, Луны и больших планет / Труды ИПА РАН, Вып. 10, Эфемеридная астрономия. М.: Институт прикладной астрономии РАН, 2004. С. 112-134.

17. Красинский Г. А., Питьева Е. В., Свешников М. Л., Свешникова Е. С. Уточнение эфемерид внутренних планет и Луны по радиолокационным, лазерным и мередианным измерениям 1961-1980 гг. // Бюлл. ИТА АН СССР, 1982. Т. 15, № 3. С. 145-163.

18. Кислик М. Д., Колюка Ю. Ф., Котельников В. А., Петров Г.М., Тихонов В. Ф. Единая релятивистская теория движения внутренних планет Солнечной системы // Докл. $A H$ СССР, 1980. Т. 255, № 3. С. 545-547; Кислик М. Д., Колюка Ю. Ф., Котельников В. А., Петров Г. М., Тихонов В. Ф. Единая релятивистская теория движения внутренних планет Солнечной системы. Релятивистские эффекты при определении орбит планет по радиолокационным наблюдениям, Научная сессия Отделения общей физики и астрономии и Отделения ядерной физики Академии наук СССР (26-27 ноября 1980 г.) // УФH, 1981. Т. 134, №1. C. 165-166. doi : 10.3367/UFNr.0134.198105j. 0165.

19. Standish E. M. JPL Planetary and Lunar Ephemerides, DE405/LE405: Jet Propulsion Laboratory Interoffice Memorandum 312.F-98-048, 1998.

20. Everhart E. Implicit single-sequence methods for integrating orbits // Celestial Mechanics, 1974. vol. 10, no. 1. pp. 35-55. doi: 10.1007/bf01261877.

21. Заусаев А. Ф., Заусаев А. А., Ольхин А. Г. Численное интегрирование уравнений движения больших планет (Меркурий-Плутон) и Луны с учетом радиолокационных наблюдений // Вестн. Сам. гос. техн. ун-та. Сер. Физ.-мат. науки, 2004. № 26. doi: $10.14498 /$ vsgtu175.

Поступила в редакцию $11 / \mathrm{XI} / 2015$;

в окончательном варианте - 21/XII/2015;

принята в печать - 26/II/2016. 
Vestn. Samar. Gos. Techn. Un-ta. Ser. Fiz.-mat. nauki

[J. Samara State Tech. Univ., Ser. Phys. \& Math. Sci.], 2016, vol. 20, no. 1, pp. 121-148

ISSN: 2310-7081 (online), 1991-8615 (print)

doi: http://dx.doi.org/10.14498/vsgtu1458

MSC: 85-08; 70M20, 65L99

\title{
COMPARISON OF THE COORDINATES OF THE MAJOR PLANETS, MOON, AND SUN OBTAINED BASED ON A NEW PRINCIPLE OF INTERACTION AND OF THE DATA BANK DE405
}

\author{
A. F. Zausaev \\ Samara State Technical University, \\ 244, Molodogvardeyskaya st., Samara, 443100, Russian Federation.
}

\begin{abstract}
In this paper the comparison of orbit coordinates and elements of large planets, the Moon and the Sun obtained on the basis of a new principle of interaction and of data bank DE405 is made. The space environment is the physical vacuum, whose properties are currently still in the formative stage. Gravity is the result of the interaction of the physical vacuum with material bodies which are moving. Gravity explains by the properties of space compression in relation to moving material bodies. Differential equations of motion of the major planets, the Moon and the Sun have been obtained. It should be noted that the system of differential equations does not contain the mass of bodies and force interactions, in addition, the Earth is considered as a spheroid. By numerical integration of the equations of motion coordinates of the Moon, the Sun and major planets osculating elements of the orbits of the inner planets during 1602-2193 are computed. The results of calculations are compared with the coordinates and orbital elements determined according to the coordinates and velocities DE405. It is shown that in contrast to Newtonian mechanics and relativistic equations of motion, the coordinates of the major planets of the Moon and the Sun, based on the solution of a new system of differential equations, are in satisfactory agreement with the coordinates of these objects obtained using data bank DE405. The resulting equations do not contain terms that take into account the non-sphericity of the Earth and the Moon, being a non-relativistic equations. Based on the research the following conclusions are made: obtained differential equations of motion satisfactorily describe the motion of the major planets, of the Moon and Sun on the time interval of 600 years; these equations are much simpler and more accurate then the differential equations that take into account the relativistic effects.
\end{abstract}

Keywords: orbital elements, numerical integration, differential equation of motion.

(C) 2016 Samara State Technical University.

Please cite this article in press as:

$\mathrm{Z}$ a usaev A. F. Comparison of the coordinates of the major planets, Moon, and Sun obtained based on a new principle of interaction and of the data bank DE405, Vestn. Samar. Gos. Tekhn. Univ., Ser. Fiz.-Mat. Nauki [J. Samara State Tech. Univ., Ser. Phys. \& Math. Sci.], 2016, vol. 20, no. 1, pp. 121-148. doi: 10.14498/vsgtu1458. (In Russian)

Author Details:

Anatoly F. Zausaev (Dr. Phys.\& Math. Sci.; zausaev_af@mail.ru), Professor, Dept. of Applied Mathematics \& Computer Science. 


\section{ORCID}

Anatoly F. Zausaev: http://orcid.org/0000-0002-5035-9615

\section{REFERENCES}

1. Chebotarev G. A. Analytical and Numerical Methods of Celestial Mechanics, American Elsevier Publishing Co., Inc., 1967, xviii+331 pp.

2. Subbotin M. F. Vvedenie $v$ teoreticheskuiu astronomiiu [Introduction to theoretical astronomy]. Moscow, Nauka, 1968, 800 pp. (In Russian)

3. Le Verrier U. J. Theorie du movement de Mercure, Annales de l'Observatoire imperial de Paris, vol. 5. Paris, Mallet-Bachelier, 1859, 195 pp.

4. Roseveare N. T. Mercury's Perihelion, from Le Verrier to Einstein. Oxford, Clarendon, 1982, $208 \mathrm{pp}$.

5. Zausaev A. F. Theory of motion of $n$ material bodies, based on a new interaction principle, Vestn. Samar. Gos. Tekhn. Univ. Ser. Fiz.-Mat. Nauki [J. Samara State Tech. Univ., Ser. Phys. \& Math. Sci.], 2006, no. 43, pp. 132-139. doi: 10.14498/vsgtu463.

6. Bogorodsky A. F. Vsemirnoe tiagotenie [Universal Gravitation]. Kiev, Naukova Dumka, 1971, 352 pp. (In Russian)

7. Brumberg V. A. Reliativistskaia nebesnaia mekhanika [Relativistic Celestial Mechanics]. Moscow, Nauka, 1972, 384 pp. (In Russian)

8. Rashevskii P. K. Rimanova geometriia i tenzornyi analiz [Riemannian geometry and tensor analysis]. Moscow, Nauka, 1967, 644 pp. (In Russian)

9. Newhall X. X., Standish E. M., Williams J. G. DE 102: A numerically integrated ephemeris of the moon and planets spanning forty-four centuries, Astronomy and Astrophysics, 1983, vol. 125, no. 1, pp. 150-167.

10. Zausaev A. F. A study of the orbital evolution of 10 short-period comets by solving differential equations of motion obtained on the basis of a new principle of interaction, Vestn. Samar. Gos. Tekhn. Univ. Ser. Fiz.-Mat. Nauki [J. Samara State Tech. Univ., Ser. Phys. \& Math. Sci.], 2007, no. 1(14), pp. 79-84 (In Russian). doi: 10.14498/vsgtu491.

11. Riemann B. Natural Philosophy, Sochineniia [Collected Works]. Moscow, Leningrad, OGIZ, GITTL, 1948, pp. 467-477 (In Russian).

12. Poincaré A. The Last Thought, $O$ nauke [On Science. Collected Works]. Moscow, Nauka, 1983, pp. 407-520 (In Russian).

13. Vizgin V. P. Relyativistskaya teoriya tyagoteniya (Istoki i formirovanie, 1900-1915) [The relativistic theory of gravitation. Sources and formation, 1900-1915]. Moscow, Nauka, 1981, 352 pp. (In Russian)

14. Zausaev A. F. The Investigation of the Motion of Planets, the Moon, and the Sun Based on a New Principle of Interaction, Vestn. Samar. Gos. Tekhn. Univ. Ser. Fiz.-Mat. Nauki [J. Samara State Tech. Univ., Ser. Phys. \& Math. Sci.], 2014, vol. 3(36), pp. 118-131 (In Russian). doi : 10.14498/vsgtu1304.

15. Zausaev A. F., Zausaev A. A. Matematicheskoe modelirovanie orbital'noi evoliutsii malykh tel Solnechnoi sistemy [Mathematical modelling of orbital evolution of small bodies of the Solar system]. Mashinostroenie-1, Moscow, 2008, 250 pp. (In Russian)

16. Pitjeva E. V. Modern Numerical Theories of the Motion of the Sun, Moon and Major Planets, Tr. Inst. Prikl. Astron. Ross. Akad. Nauk, 2004, vol. 10, pp. 112-134 (In Russian).

17. Krasinskii G. A., Piteva E. V., Sveshnikov M. L., Sveshnikova E. S. Improvement of the ephemerides of the inner planets and the moon using radar, laser, and meridian measurements during 1961-1980, Institut Teoreticheskoi Astronomii, Biulleten', 1982, vol. 15, no. 3, pp. 145-164 (In Russian).

18. Kislik M. D., Kolyuka Yu. F., Kotel'nikov V. A., Tikhonov V. F. A Unified Relativistic Theory of the Motion of the Inner Planets of the Solar System, Proceedings of the USSR Academy of Sciences, 1980, vol. 255, no. 3, pp. 545-547 (In Russian); Kislik M. D., Kolyuka Yu. F., Kotel'nikov V. A., Tikhonov V. F. A Unified Relativistic Theory of the 
Motion of the Inner Planets of the Solar System. Relativistic Effects in Determination of the Orbits of the Planets from Radar Observations, Sov. Phys. Usp., 1981, vol. 24, no. 1, pp. 437-438. doi: 10.1070/PU1981v024n05ABEH004807.

19. Standish E. M. JPL Planetary and Lunar Ephemerides, DE405/LE405, Jet Propulsion Laboratory Interoffice Memorandum 312.F-98-048, 1998.

20. Everhart E. Implicit single-sequence methods for integrating orbits, Celestial Mechanics, 1974, vol. 10, no. 1, pp. 35-55. doi: 10.1007/bf01261877.

21. Zausaev A. F., Zausaev A. A., Ol'khin A. G. The numerical integration of the equations of motion for large planets (Mercury and Pluto) and the Moon with the radar observations, Vestn. Samar. Gos. Tekhn. Univ. Ser. Fiz.-Mat. Nauki [J. Samara State Tech. Univ., Ser. Phys. \& Math. Sci.], 2004, no. 26, pp. 43-47 (In Russian). doi : 10.14498/vsgtu175.

Received 11/XI/2015;

received in revised form $21 / \mathrm{XII} / 2015$;

accepted 26/II/2016. 